\title{
Early pathogenic events associated with Sjögren's syndrome (SjS)-like disease of the nod mouse using microarray analysis
}

\author{
Smruti Y Killedar ${ }^{1, *}$, Sarah E Eckenrode ${ }^{2}$, Richard A McIndoe ${ }^{2}$, Jin-Xiong She ${ }^{2}$, \\ Cuong Q Nguyen ${ }^{1}$, Ammon B Peck ${ }^{1,3,4}$ and Seunghee R Cha ${ }^{5}$ \\ ${ }^{1}$ Department of Oral Biology, College of Dentistry, University of Florida, Gainesville, FL, USA; \\ ${ }^{2}$ Center for Biotechnology \& Genomic Medicine, Medical College of Georgia, Augusta, GA, USA; \\ ${ }^{3}$ Department of Pathology, Immunology \& Laboratory Medicine, College of Medicine, University of Florida, \\ Gainesville, FL USA; ${ }^{4}$ Center for Orphan Autoimmune Diseases, College of Dentistry, University of Florida, \\ Gainesville, FL, USA and ${ }^{5}$ Department of Oral \& Maxillofacial Surgery, College of Dentistry, University of \\ Florida, Gainesville, FL, USA
}

\begin{abstract}
Recently, we reported development of the C57BL/6.NOD-Aec1Aec2 mouse carrying two genetic intervals derived from the NOD mouse. These two genetic regions confer full Sjögren's syndrome (SjS)-like disease in SjS-non-susceptible C57BL/6 mice. The current study was undertaken to apply microarray technology to define the molecular basis underlying onset of SjS-disease in C57BL/6.NOD-Aec1Aec2 mice. Using oligonucleotide microarrays, gene expression profiles of submandibular glands derived from 8- to 12-week-old C57BL/6.NODAec1Aec2 mice and 8-week-old C57BL/6 mice were performed for comparison. Significant differential expressions were determined using the Mann-Whitney $U$ test. Hybridizations using submandibular cDNA probes revealed 75 differentially expressed genes at 8 weeks and 105 differentially expressed genes at 12 weeks of age in C57BL/6.NOD-Aec1Aec2 mice compared to 8-week-old C57BL/6 mice. These genes were related generally to basic cellular activities such as transcription, translation, DNA replication, and protein folding. During the predisease phase, genes upregulated encode proteins associated with the IFN-gamma signaltransduction-pathway (Jak/Stat1), TLR-3 (Irf3 and Traf6) and apoptosis (casp11 and casp3), indicative of chronic proinflammatory stimuli, especially IL-1. Between 8 and 12 weeks of age, sets of clustered genes were upregulated that are associated with adaptive immune responses, especially $B$ cell activation, proliferation and differentiation (Baffr, Taci, Bcma, Blys, April, CD70, CD40L, Traf1, Traf3, Pax5, c-Jun, Elk1 and Nf-kB), and neural receptors (Taj/Troy). Altered gene expressions of TLR3 and TNF-superfamily-receptors and ligands during this early phase of SjS suggest a possible viral etiology in the altered glandular homeostasis with an upregulated, possibly overstimulated, B-lymphocyte activation in the early autoimmune response present in the submandibular glands. The importance of NF- $\kappa \mathrm{B}$ as a critical signal transduction pathway is also suggested but its link is not yet clear.
\end{abstract}

Laboratory Investigation (2006) 86, 1243-1260. doi:10.1038/labinvest.3700487; published online 30 October 2006

Keywords: Sjögren's syndrome; microarray; autoimmunity; NOD mouse; salivary gland

Sjögren's syndrome $(\mathrm{SjS})$ is an autoimmune disease initially targeting primarily the salivary and lacrimal glands, resulting in dry mouth (xerostomia sicca) and/or dry eye (keratoconjunctivitis sicca) disease,

Correspondence: Professor SR Cha, DDS, PhD, Department of Oral \& Maxillofacial Surgery, College of Dentistry, University of Florida, PO Box 100416, Gainesville, FL 32610, USA.

E-mail: SCHA@dental.ufl.edu

${ }^{*}$ Current address: The Johns Hopkins Asthma \& Allergy Center, 5501 Hopkins Bayview Circle, Rm 2A 58, Baltimore, MD 21224, USA

Received 2 August 2006; revised and accepted 14 September 2006; published online 30 October 2006 respectively. ${ }^{1}$ While numerous mouse strains have been developed to study SjS, the NOD mouse has become one of the more extensively characterized for investigating the pathogenesis of autoimmune exocrinopathy. ${ }^{2-8}$ Our analyses indicate that NOD mice exhibit loss of saliva flow (up to $75 \%$ ) and tear flow (up to $35 \%$ ) by 16-24 weeks of age ${ }^{9,10}$ concomitant with leukocyte infiltration of the glands, and this contrasts with other strains such as the NFS/sld mutant mouse thymectomized 3 days after birth or the BAFF transgenic mouse which exhibit salivary dysfunction at much older ages (Ishimaru et al, ${ }^{11}$ Groom et $a l,{ }^{12}$ respectively) and 
often independently of leukocyte infiltration of the exocrine glands. In addition, NOD mice undergo multiple immunopathological changes between 12 and 20 weeks of age, including increases in proinflammatory cytokines, autoantibody production, and levels of acinar cell apoptosis, which closely resemble the disease characteristics in human patients..$^{9,10,12}$

Interestingly, these pathological changes observed in NOD mice appear to occur as a consequence of altered glandular homeostasis. ${ }^{13,14}$ Aberrant proteolytic activity, elevated apoptosis, downregulated epidermal growth factor (EGF) gene expression, and reduced amylase activity are commonly observed in NOD mice prior to or during disease onset around 8-10 weeks of age. Although what drives these alterations remains unknown, this altered glandular homeostasis could explain why autoreactive T-cells develop responsiveness against exocrine gland cells in this systemic autoimmune disorder. ${ }^{7,13}$ Thus, the NOD mouse model of SjS has given rise to the concept that autoimmune exocrinopathy develops in two phases. ${ }^{3,5,7,13-17}$ The initial phase is lymphocyte-independent and occurs as a consequence of intrinsic problems in exocrine tissue homeostasis or differentiated function, potentially associated with delayed-expression of self-antigens or exposure of cryptic/neo antigens. The second phase involves a subsequent tissue specific immunological attack by activated T-cells, B-cells and their products against corresponding self-antigens that leads to SjS-like autoimmune exocrinopathy in the target organs. ${ }^{18-20}$

Studies using NOD mice, considered the best animal model of type I diabetes (T1D), have identified several diabetogenic genetic regions on different chromosomes, referred to as Idd susceptibility genes, that determine genetic susceptibility to development of T1D. ${ }^{21,22}$ Our previous studies that screened for Idd genes responsible for development of SjS-like disease in NOD mice indicated that two genetic intervals, one containing the Idd5 locus on chromosome 1 and the other containing the Idd3 locus on chromosome 3 , control the physiological aberrations and immunological responses, respectively. ${ }^{2,23}$ At the same time, no role for Idd1, containing the MHC locus $H-2^{g 7}$ could be identified. Subsequent studies showed that Idd3 and Idd5, when placed into C57BL/6 mice, were both necessary and sufficient to permit full development of a SjS-like disease. This new SjS-susceptible strain is designated C57BL/6.NOD-Aec1Aec2, where Aec1 corresponds to Idd 3 and Aec2 corresponds to Idd $5 .^{23}$

Our previous studies defining the SjS-like disease in NOD mice have focused primarily on the role of individual molecules through the use of gene knockout (KO) mice. While the information obtained from such experiments is ideal for uncovering possible roles of these individual molecules in the disease process, such an approach is less than optimal for discovering new genes or molecular networks involved in the development and onset of disease. In the present studies, we have taken advantage of microarray technology to screen large numbers of genes in order to identify those that are differentially expressed temporally in submandibular glands of the newly generated C57BL/6.NODAec1Aec2 mouse relative to the nonautoimmune C57BL/6 parental mouse. Use of C57BL/6.NODAec1Aec2 mice permits comparison with its parental, non-diseased C57BL/6 mice, thereby eliminating differentially expressed genes due to very different genetic backgrounds, for example, in a comparison of NOD vs a control strain.

\section{Materials and methods}

\section{Animals}

C57BL/6J and C57BL/6.NOD-Aec1Aec2 mice were bred and maintained under SPF conditions within Animal Care Services at the University of Florida, Gainesville. The animals were maintained on a $12 \mathrm{~h}$ light-dark schedule and provided water and food $a d$ libitum. For this study, female mice were euthanized at either 8 or 12 weeks of age. Both breeding and use of these animals were approved by the University of Florida, IACUC.

\section{Detection of Differentially Expressed Genes using Microarray Analyses}

Hybridizations were carried out using MWG Mouse $30 \mathrm{~K}$ A Arrays (MWG Biotech, NC, USA) containing 10752 genes. Microarray slides were produced inhouse using epoxy-coated slides printed by the MicroGrid TAS II Biorobotics system (Genetix USA Inc., Boston, MA, USA). Total RNA was isolated from the submandibular glands freshly extracted from individual C57BL/6.NOD-Aec1Aec2 $(n=5)$ and C57BL/6J $(n=5)$ mice at either 8 or 12 weeks of age using the RNeasy Mini-Kit (Qiagen, Valencia, CA, USA), as per the manufacturer's protocol. Reference RNA was prepared by combining equal quantities of total RNA from the submandibular glands of C57BL/6J mice $(n=8)$ at 8 weeks of age. The 3DNA Array 350kit (Genisphere Inc., Hatfield, PA, USA) was then used to create the hybridization probes from $500 \mathrm{ng}$ of total RNA for each C57BL/ 6.NOD-Aec1Aec2 mouse (labeled with Cy3) and each parental C57BL/6J mouse (labeled with Cy5). Each Cy3-labeled sample probe was combined with an equal quantity of the Cy5-labeled reference probe and hybridized to a printed array for $16 \mathrm{~h}$ at $42^{\circ} \mathrm{C}$. The slides were then washed, dried by centrifugation, and placed in the dark to avoid exposure to light until scanned. Hybridizations were repeated three times for each RNA sample to improve the accuracy of the measurements. 


\section{Data Acquisition}

An Affymetrix $428^{\mathrm{TM}}$ Scanner (Affymetrix, Santa Clara, CA, USA) was used to create the images by scanning the slide twice, the first time at $532 \mathrm{~nm}$ and the second time at $635 \mathrm{~nm}$. This process generated two 16-bit tagged image file format (TIFF) image files. Numerical values for each spot were extracted from the images using MolecularWare (MolecularWare, Cambridge, MA, USA). A statistical program was used to identify/flag spots with low-intensity/ background ratios. This flagging procedure allowed, first, a determination of the data quality for each spot (gene) as being sufficiently good to warrant subsequent analysis, and second, elimination of unreliable elements with expression statistically too similar to the background. After 'flagging', the data were uploaded to a flat file database, where the gene expression information was linked to the coordinates of the spot on the array.

\section{Statistical Analyses}

For each RNA sample, the medians of the replicates were determined and used to determine the ratios of expression $\left(\log _{2}\right)$. Subsequent statistical analyses were based on these median values. The Student's $t$ test was used to identify genes with the highest discrimination power between the comparative groups. On first pass, genes were considered differentially expressed if the $P$-value $<0.0001$. Genes of interest were clustered using Cluster software and viewed using TreeView. These microarray data were analyzed further using a nonparametric Mann-Whitney $U$ test (Statistica, Tulsa, OK, USA) in order to identify genes that are statistically different between the C57BL/6.NOD-Aec1Aec2 and C57BL/6 with a $P$-value $<0.01$.

\section{Verification of Selected Genes by Semiquantitative RT-PCR}

Total RNA was prepared from freshly explanted submandibular glands using the Rneazy Mini Kit (Qiagen, Valencia, CA, USA). cDNA was synthesized using $4 \mu \mathrm{g}$ of total RNA, Superscript II reverse transcriptase (Invitrogen Life Technologies, Carlsbad, CA, USA), and pd(T)12-18 oligomeric DNA (Amersham Pharmacia, Piscataway, NJ, USA). The cDNA was quantified by spectrophotometry. Semiquantitative PCRs were carried out using $1 \mu \mathrm{g}$ of cDNA as template. Following an initial denaturation at $94^{\circ} \mathrm{C}$ for $4 \mathrm{~min}$, each PCR was carried out for 40 cycles consisting of $94^{\circ} \mathrm{C}$ for $1 \mathrm{~min}$, optimal annealing temperature for $45 \mathrm{~s}$ and $72{ }^{\circ} \mathrm{C}$ for $2 \mathrm{~min}$. The forward and reverse sequences of each primer set are:

p38-forward: $\quad$ 5'-TCA ACC AGG AAG TGA GTG GCT GAA-3' p38-reverse:

Ch1-forward:

Ch1-reverse:

Adh5-forward:

Adh5-reverse:

B18-forward:

B18-reverse:

Pfdn5-forward:

Pfdn5-reverse:

Stat-forward:

Stat-reverse:

Pde4-forward:

Pde4-reverse:

Rac1-forward:

Rac1-reverse:

Map2k4-forward:

Map2k4-reverse:

Jnk-forward:

Jnk-reverse:

$N f k b$-forward:

Nfkb-reverse:

Traf6-forward:

Traf6-reverse:

BAFFR-2-forward:

BAFFR-2-reverse:

Baff-forward:

Baff-reverse:

$\beta$-Actin-forward:

$\beta$-Actin-reverse:

PCR products were size separated by electrophoresis using a $0.9 \%$ agarose gels and visualized
5'-ATC TTG GCA CCT CTC AGA GCC TTT-3'

5'-AGC CAG ATC TGA AGC ACG TGA AGA-3'

5'-AGT GCTTCG TGA AGG GTC TCC AAT-3'

$5^{\prime}$-ATC TTG GGA CAT GAA GGT GCT GGA-3'

5'-ACG TTG CCA ATG CAC TCA AAG GAG-3'

5'-GCA GCA GGG CAC CGT GAC AAA G-3'

5'-ACG CAG GAG GGC ATC AAA GAG CA-3'

5'-GGC CCA CAT TTG GGT GGA AGA TTT-3'

5'-CGT TGCTCT TGT TCA GCA CGT TCA-3'

5'-TGGG GGA GGG GCC TTC TTG ATG-3'

5'-TGG CCC CCT TAA TGG ATG TGC AA-3'

5'-CCC GCG TCA GTG CCT TTG CTA T-3'

5'-CGG CGC TCC ATG AAG GTT CGT-3'

5'-TGG GTG TGC TGG GTG GAG TGT GA-3'

5'-TGG GGA GGG ACG GCA GTG GAG-3'

5'-GCG ATG TGC TCA GCC AAA TTC CC-3'

5'-CCT GGC CCA TGA TGT CGA GAA GC-3'

5'-AGC TCG GAA CAC CTT

GTC CTG AAT-3'

5'-AGC CAT TGA TCA TTG CTG CAC CTG-3'

5'-TTC TGC ATG GCG ATG TCA AAG CTG-3'

5'-ATG CTG AGG CAG GAG AGG ATT TGT-3'

5'-GCA CAA GTG CCC AGT TGA CAA TGA-3'

5'-AGT GTC GTG CCA AGT GAT TCC TCT-3'

5'-AGC AGT TCG GTT TGT GTG CTG TTC-3'

5'-CCG CAG TGC ATT CTG GGA ATC AAA-3'

5'-AACGGAGACGACACCTTC TTTGGT-3'

5'-CTGAACATGTGTCACCCA AGGCAA-3'

5'-CCT GAA CCC TAA GGC CAA CCG-3'

5'-GCT CAT AGCTCT TCTCCA GGG-3'

Laboratory Investigation (2006) 86, 1243-1260 
with ethidium bromide staining. PCR band intensities were compared to $\beta$-actin using the Flourchem Imaging densitometer system (Alpha Innotech Corporation, San Leandro, CA, USA). Relative band intensities were determined by dividing the intensity of selected genes mRNA by the density of $\beta$-actin band.

\section{Results}

Differential Gene Expression in the Submandibular Glands of 8-week-old C57BL/6. NOD-Aec1Aec2 Mice Normalized to 8-week-old C57BL/6J Mice

C57BL/6.NOD-Aec1Aec2 mice exhibit both the pathophysiological characteristics and reduced secretory responses observed with NOD mice; however, the disease process appears to be accelerated in the C57BL/6.NOD-Aec1Aec2 mouse suggesting either the genetic background of C57BL/6J contributes positively to the disease or the genetic background of NOD contributes some resistance genes. $^{2,23}$ The present study was designed to define gene expression profiles within the submandibular glands of C57BL/6.NOD-Aec1Aec2 mice at two time points, 8 weeks of age (representing an early preclinical phase of disease) and 12 weeks of age (representing the early clinical phase of autoimmunity). By examining these two time points, genes identified as being differentially expressed may be correlated with one or more manifestations of abnormal glandular homeostasis and the initiation of the autoimmune response, thus enabling us to separate and dissect the pathophysiological vs immunological aspects of the SjS-like disease. The use of the C57BL/6J and C57BL/6.NOD-Aec1Aec2 combination permits four comparisons: (1) C57BL/ 6J (8 weeks of age) vs C57BL/6J (12 weeks of age) showing normal age-related changes in gene expressions, (2) C57BL/6J (8 weeks of age) vs C57BL/ 6.NOD-Aec1Aec2 (8 weeks of age) showing early phase disease-related gene expressions, (3) C57BL/ 6J (12 weeks of age) vs C57BL/6.NOD-Aec1Aec2 (12 weeks of age) showing late phase disease-related gene expressions, and (4) C57BL/6J (8 weeks of age) vs C57BL/6.NOD-Aec1Aec2 (12 weeks of age) showing changes in both age- and disease-initiating gene expressions. For the current study, the data from (2) and (4) are presented to focus on identification of possible underlying mechanisms for onset of SjS-like disease. We have utilized oligonucleotide microarrays, as opposed to cDNA arrays, making identification of differentially expressed genes faster, although recognizing that organ-specific genes may be missed.

A comparison of differentially expressed genes in the submandibular glands of C57BL/6.NODAec1Aec2 vs C57BL/6J mice at 8 weeks of age (a time at which early preclinical manifestations should be evident and earliest infiltrations of leukocytes be present) identified 75 genes reaching statistical significance from the profiling of 10752 genes. The expression profiles of these 75 genes are compared in Figure 1 between 5 individual samples from each strain, revealing the consistent differential
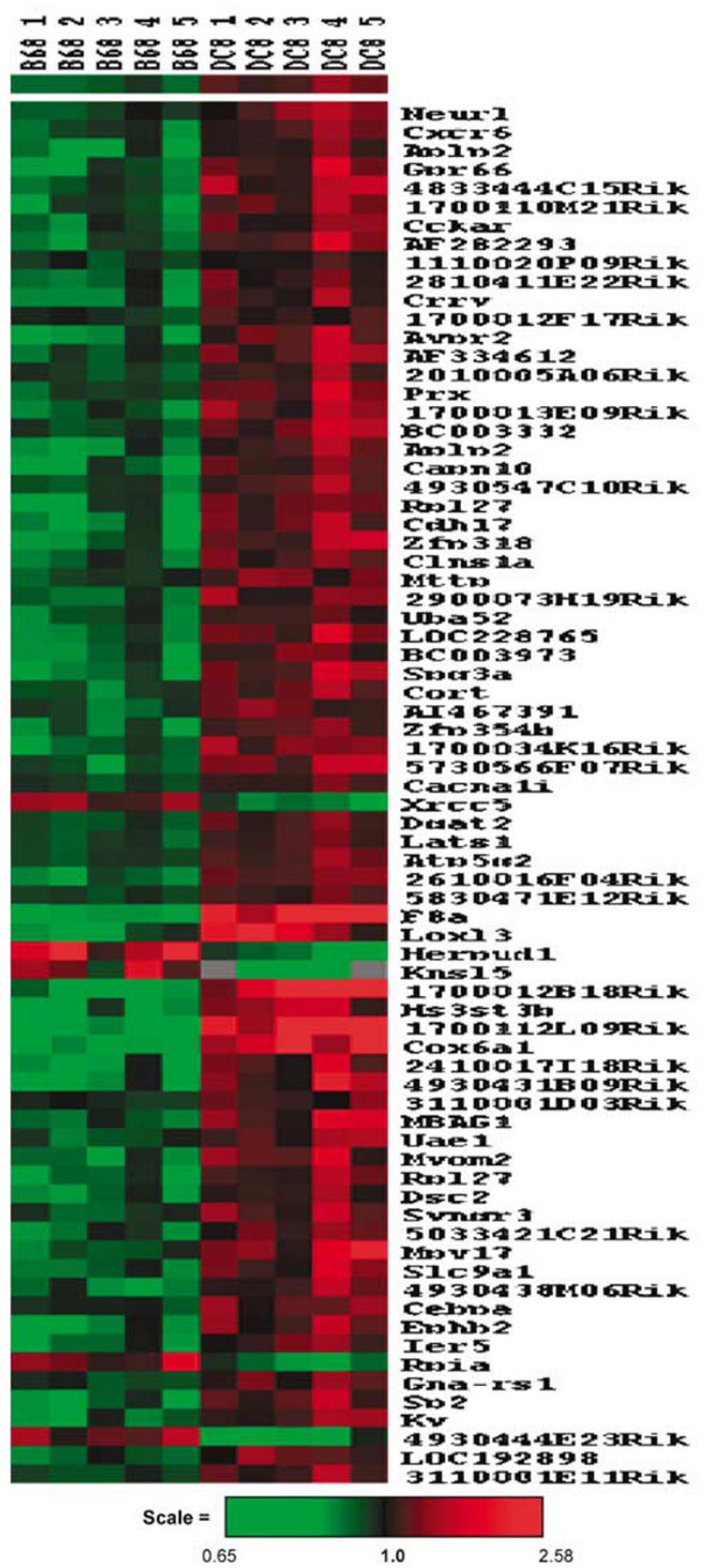

Figure 1 TreeView map of differentially expressed genes in the submandibular glands of 8-week-old C57BL/6.NOD-Aec1Aec2 mice compared to those of 8-week-old C57BL/6 mice. cDNA from the submandibular glands of individual mice $(n=5$ per group) were hybridized against a partial genomic oligonucleotide library (MWG Mouse 30 K A Arrays, MWG Biotech) and further analyzed for significant differences as determined by the Mann-Whitney $U$ test (see Table 1). Upregulated genes are shown in red, while downregulated genes are shown in green. 
expressions among the individual mice within each group. Of the 75 differentially expressed genes identified in this first analysis, 71 were upregulated and four downregulated in the C57BL/ 6.NOD-Aec1Aec2 mice compared to the parental C57BL/6 mice. The mean expression levels for these 75 genes in the C57BL/6.NOD-Aec1Aec2 mice normalized against the mean expression levels in the C57BL/6J mice are presented in Table 1 together with known biological functions. As might be expected, these genes are involved in multiple cellular functions, including DNA replication, transcription and translation, signal transduction and protein activation, as well as ion transport, metabolism and energy production. In addition, a number of genes encode membraneassociated proteins, while the functions of 15 genes remain unknown. Of particular interest is the fact that five genes are located on chromosome 1 (ie, Ier5, Capn10, Xrcc5, Crry and Gpr66) and two genes on chromosome 3 (ie, 4930431B09Rik and 4930444E23Rik).

Table 1 Relative differential gene expressions in submandibular glands from 8-week-old C57BL/6 mice vs 8-week-old C57BL/6.NODAec1Aec2 mice, as determined by the Mann-Whitney $U$ test

\begin{tabular}{|c|c|c|c|c|c|}
\hline Gene & Gene description & $\begin{array}{c}B 6: D C \\
(8 \text { weeks })\end{array}$ & P-value & $\begin{array}{c}B 6: D C \\
(12 \text { weeks) }\end{array}$ & $\mathrm{P}$-value \\
\hline
\end{tabular}

Signal transduction

Cxcr6

Cckar

Syngr3

Myom2

MBAG1

Neurl

Avpr2

Prx

AF334612

1700112L09Rik

LOC228765

3110001E11Rik

4930438M06Rik

Transcription

F8a

Zfp354b

Zfp318

LOC192898

BC003332

Sp2

2010005A06Rik

4930547C10Rik

2610016F04Rik

Cell cycle

5730566F07Rik

Ier5

Carbohydrate Rpia

Lipid

Uae1

Protein

Hs3st3b

Herpud1

Cort

Rpl27

Uba52

2900073H19Rik

Gpr66

Cadherin associated

AI467391

Cdh17

2010005A06Rik
Chemokine (C-X-C motif) receptor 6

Cholecystokinin A receptor

Synaptogyrin 3

Myomesin 2

Leucine rich repeat containing 4

Neuralized homolog (Drosophila)

Arginine vasopressin receptor 2

Periaxin

PDZ domain containing 2

Chimerin

Syndecan-binding protein (syntenin) 2

RIKEN cDNA 3110001E11 gene

RIKEN cDNA 4930438M06 gene

Factor 8-associated gene A

Zinc-finger protein 354B

Zinc-finger protein 318

Zinc-finger CCCH type domain containing 5

cDNA sequence BC003332

Sp2 transcription factor

RIKEN cDNA 2010005A06 gene

RIKEN cDNA 4930547C10 gene

RIKEN cDNA 2610016F04 gene

RIKEN cDNA 5730566F07 gene

Immediate early response 5

1.38

1.29

$<0.010$

$<0.010$

0.56

1.27

$<0.010$

$<0.010$

$<0.010$

$<0.010$

$<0.010$

$<0.010$

$<0.010$

$<0.010$

0.73

$<0.010$

0.74

$<0.010$

Glucosamine

1.26

$<0.010$

1.50

$<0.010$

Heparan sulfate (glucosamine) 3-O-sulfotransferase 3B

Homocysteine-inducible, endoplasmic reticulum

stress-inducible, ubiquitin-like

Cortistatin

Ribosomal protein L27

1.52

0.60

$<0.010$

$<0.010$

0.61
1.09

$<0.010$

$<0.010$

1.17

1.35

Ubiquitin A-52 residue ribosomal protein fusion product 1

RIKEN cDNA 2900073H19 gene

$\mathrm{G}$ protein-coupled receptor 66

1.37

$<0.010$

$<0.010$

1.12

1.12

1.24

$<0.010$

1.22

$<0.010$

Casitas B-lineage lymphoma-like 1

Cadherin 17

RIKEN cDNA 2010005A06 gene
1.25

1.41

1.22
$<0.010$

$<0.010$
$<0.010$

NS

NS

$<0.010$

NS

0.046

0.016

$<0.010$

$<0.010$

$<0.010$

NS

$<0.010$
NS

$<0.010$

$<0.010$

NS

$<0.010$

NS

NS

NS

NS

NS

.

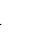

.

.


Table 1 Continued

\begin{tabular}{|c|c|c|c|c|c|}
\hline Gene & Gene description & $\begin{array}{c}B 6: D C \\
(8 \text { weeks })\end{array}$ & $\mathrm{P}$-value & $\begin{array}{c}B 6: D C \\
(12 \text { weeks })\end{array}$ & P-value \\
\hline \multicolumn{6}{|l|}{ Immune system } \\
\hline Crry & Complement receptor-related protein & 1.32 & $<0.010$ & 1.26 & 0.026 \\
\hline \multicolumn{6}{|l|}{ Energy production } \\
\hline Atp5g2 & ATP synthase, $\mathrm{H}+$ transporting, mitochondrial & 1.23 & $<0.010$ & 1.19 & NS \\
\hline Knsl5 & ATP-binding & 0.41 & $<0.010$ & 0.48 & $<0.010$ \\
\hline BC003973 & lysyl oxidase-like 1 & 1.31 & $<0.010$ & 1.15 & 0.012 \\
\hline \multicolumn{6}{|l|}{ Protein kinase } \\
\hline Lats1 & Large tumor suppressor & 1.21 & $<0.010$ & 1.11 & NS \\
\hline Aplp2 & Amyloid beta (A4) precursor-like protein 2 & 1.34 & $<0.010$ & 1.26 & NS \\
\hline 2810411E22Rik & RIKEN cDNA 2810411E22 & 1.26 & $<0.010$ & 1.43 & 0.021 \\
\hline Ephb2 & Eph receptor B2 & 1.38 & $<0.010$ & 1.23 & NS \\
\hline Xrcc5 & $\mathrm{X}$-ray repair complementing defective repair in $\mathrm{CH}$ cells 5 & 0.72 & $<0.010$ & 1.08 & NS \\
\hline Capn10 & Calpain 10 & 1.38 & $<0.010$ & 1.18 & NS \\
\hline Ky & Kyphoscoliosis & 1.32 & $<0.010$ & 1.12 & NS \\
\hline Cebpa & CCAAT/enhancer-binding protein (C/EBP), alpha & 1.21 & $<0.010$ & 0.99 & NS \\
\hline \multicolumn{6}{|c|}{ Ion channel/transport } \\
\hline Clns1a & Chloride channel, nucleotide-sensitive, 1A & 1.27 & $<0.010$ & 1.30 & NS \\
\hline Slc9a1 & $\begin{array}{l}\text { Solute carrier family } 9 \text { (sodium/ hydrogen exchanger), } \\
\text { member } 1\end{array}$ & 1.33 & $<0.010$ & 1.23 & NS \\
\hline Cacna1i & Calcium channel, alpha 1I subunit & 1.18 & $<0.010$ & 0.96 & NS \\
\hline 2010005A06Rik & RIKEN cDNA 2010005A06 gene & 1.22 & $<0.010$ & 1.04 & NS \\
\hline \multicolumn{6}{|l|}{ Membrane protein } \\
\hline Cox6a1 & Cytochrome $c$ oxidase, subunit VI a, polypeptide 1 & 1.83 & $<0.010$ & 4.93 & $<0.010$ \\
\hline Dgat2 & Diacylglycerol $O$-acyltransferase 2 & 1.23 & $<0.010$ & 1.26 & 0.027 \\
\hline Spg3a & Spastic paraplegia 3A homolog (human) & 1.44 & $<0.010$ & 1.52 & $<0.010$ \\
\hline AF282293 & Olfactory receptor 971 & 1.34 & $<0.010$ & 1.27 & 0.044 \\
\hline Gna-rs1 & Guanine nucleotide-binding protein, related sequence 1 & 1.21 & $<0.010$ & 1.42 & $<0.010$ \\
\hline Mpv17 & Mpv17 transgene, kidney disease mutant & 1.38 & $<0.010$ & 1.39 & $<0.010$ \\
\hline 3110001D03Rik & RIKEN cDNA 3110001D03 gene & 1.16 & $<0.010$ & 1.10 & $<0.010$ \\
\hline Dsc2 & Desmocollin 2 & 1.28 & $<0.010$ & 1.17 & NS \\
\hline \multicolumn{6}{|l|}{ Unknown function } \\
\hline Loxl3 & lysyl oxidase-like 3 & 1.62 & $<0.010$ & 2.28 & $<0.010$ \\
\hline 1700013E09Rik & RIKEN cDNA 1700013E09 gene & 1.34 & $<0.010$ & 1.22 & $<0.010$ \\
\hline 2410017I18Rik & RIKEN cDNA 2410017 I18 gene & 1.41 & $<0.010$ & 1.45 & $<0.010$ \\
\hline AF282293 & Olfr971-olfactory receptor 971 & 1.34 & $<0.010$ & 1.27 & 0.044 \\
\hline 5830471E12Rik & RIKEN cDNA 5830471E12 gene & 1.19 & $<0.010$ & 1.13 & NS \\
\hline 1700034K16Rik & RIKEN cDNA 1700034K16 gene & 1.36 & $<0.010$ & 1.23 & 0.076 \\
\hline BC003332 & cDNA sequence BC003332 & 1.29 & $<0.010$ & 1.14 & NS \\
\hline 1700013E09Rik & RIKEN cDNA 1700013E09 gene & 1.34 & $<0.010$ & 1.46 & $<0.010$ \\
\hline 4930444E23Rik & RIKEN cDNA 4930444E23 gene & 0.65 & $<0.010$ & 0.56 & $<0.010$ \\
\hline 1700012F17Rik & RIKEN cDNA 1700012 F17 gene & 1.14 & $<0.010$ & 1.09 & 0.046 \\
\hline 1110020P09Rik & RIKEN cDNA 1110020P09 gene & 1.13 & $<0.010$ & 1.10 & NS \\
\hline 1700110M21Rik & RIKEN cDNA 1700110M21 gene & 1.32 & $<0.010$ & 1.22 & 0.012 \\
\hline 4833444C15Rik & RIKEN cDNA 4833444C15 gene & 1.34 & $<0.010$ & 1.20 & NS \\
\hline 5033421C21Rik & RIKEN cDNA 5033421C21 gene & 1.28 & $<0.010$ & 1.29 & 0.028 \\
\hline 4930431B09Rik & RIKEN cDNA 4930431B09 gene & 1.46 & $<0.010$ & 1.35 & 0.028 \\
\hline
\end{tabular}

A closer look at the genes that are differentially expressed at 8 week of age in the submandibular glands of C57BL/6.NOD.Aec1Aec2 mice tend to link membrane proteins involved in signal transductions with intracellular trafficking, especially of vesicles. Genes that encode membrane proteins include Dgat2, Spg3a, AF282293, Gna-rs1, Mpv17, 1700042F02Rik, 3110001D03Rik, and Dsc2, and these, in turn, interact with various intracellular molecules, for example, G-proteins, Cckar, 1700112l09Rik, 2010005A06Rik, Gpr66, Gna-rs1 and Spg3a, which act as chaperones and affect the activity of other molecules. The ion channel molecules such as Cns1a, Slc9a1, AF334612 which are involved in the trafficking of the vesicles, apical sorting of the proteins and subsequent degradation are also upregulated. This is supported by the upregulation of CCKar, AF334612, Synaptogyrins and molecules like Ranbp2 and Cacna1i affecting exocrine secretion and calcium channels, respectively, potentially altering the vesicle transport and/ or saliva secretion. Additionally, calcium is known 
to be important for the functions of cadherins. Removal of calcium reduces adhesive activity and renders cadherins vulnerable to proteases. As cadherins are critical to the maintenance of proper cell-cell contacts, they are important regulators of morphogenesis. ${ }^{24}$ Two genes that regulate expression of cadherin molecules (Cdh17, AI467391 and Dsc2) were also found to be significantly upregulated.

Differential Gene Expression in the Submandibular Glands of 12-week-old C57BL/6.NOD-Aec1Aec2 Mice Normalized to 8-week-old C57BL/6J Mice

A comparison of differentially expressed genes in the submandibular glands of C57BL/6.NODAec1Aec2 at 12 weeks of age (a time of detectable clinical manifestations characterized by small areas of leukocyte infiltrates) vs C57BL/6J mice at 8 weeks identified 105 genes that reached statistical significance in the profiling of the same 10752 genes analyzed above. The expression profile of these 105 differentially expressed genes is presented in Figure 2 , again revealing a consistent differential expression among the individual mice within each group. Of the 105 genes, 85 were upregulated and 20 downregulated. A listing of the known biological functions of these 105 genes is presented in Table 2 . These genes encode molecules involved in DNA replication, transcription and translation, protein folding and transport, signal transduction and protein activation, as well as ion transport, metabolism and energy production. In addition, a number of genes encode membrane-associated proteins and proteins involved in intracellular trafficking, ribosomal biogenesis, free-radical neutralization, and immune regulation. The functions of some 25 genes remain unknown. Of the differentially expressed genes, five are located on chromosome 1 (ie, Stx6, Rpl37a, Cdh74921511D23Rik, 1190006A08Rik and 1500032H18Rik) and four on chromosome 3 (ie, H2afz, Adh5, 6330415M09Rik and 4930415G15Rik).

As might be expected from the known pathophysiological analyses of C57BL/6.NOD-Aec1Aec2 mice, several of the genes which were significantly upregulated are associated with either programmed cell death or immune functions. Genes Ppard, Gabrr2, Gabrb2, Pde4b, F2rl1, B2m, Mtcp, Cd5, 2410003B16Rik, 2310043N20Rik, Jcam2, Fut8 and $N f k b i b$ certainly can affect cellular homeostasis through various chemokines, TNF- $\alpha$ responses or MAP kinases eventually leading to apoptosis of the cells. Another group of overexpressed genes includes Synaptogyrins, Synt6 Gabrb2, Cdh7, 1700012B18Rik, Atp8a1 and Aqp2 each associated with intracellular trafficking and vesicular transport through ion channels. These genes can regulate the movement of vesicles from the basal lateral to the apical membrane and possibly contribute to the flow

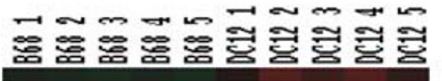

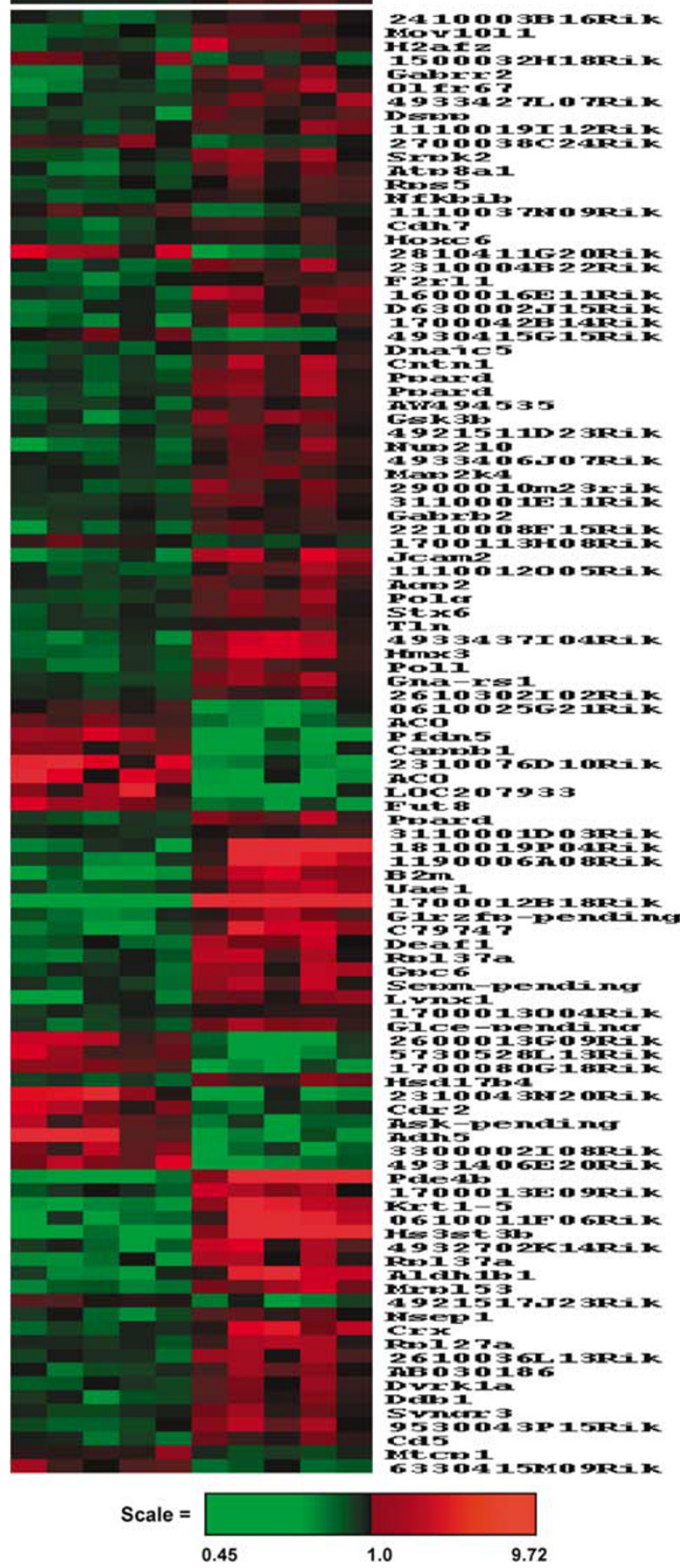

Figure 2 TreeView map of differentially expressed genes in the submandibular glands of 12-week-old C57BL/6.NOD-Aec1Aec2 mice compared to those of 8 -week-old C57BL/6 mice. cDNA from the submandibular glands of individual mice ( $n=5$ per group) were hybridized against a partial genomic oligonucleotide library and further analyzed as in Figure 1. Upregulated genes are shown in red, while downregulated genes are shown in green.

rates of saliva. Interestingly, a similar comparison for differentially expressed genes in the submandibular glands of 12-week-old vs 8-week-old C57BL/6J 
Table 2 Relative differential gene expressions in submandibular glands from 8-week-old C57BL/6 mice vs 12-week-old C57BL/6.NODAec1Aec2 mice, as determined by the Mann-Whitney $U$ test

\begin{tabular}{cccc}
\hline Gene $\quad$ Gene description & B6:DC & P-value & B6:DC \\
$(8$ weeks $)$ & P-value
\end{tabular}

Signal transduction

Ppard

Gabrr2

Gabrb2

Pde4b

F2rl1

4921511D23Rik

Cntn1

Peroxisome proliferator activator receptor delta

Gamma-aminobutyric acid (GABA-C) receptor, subunit rho-2

Gamma-aminobutyric acid (GABA-A) receptor, beta 2

Phosphodiesterase 4B, cAMP specific

Coagulation factor II (thrombin) receptor-like 1

RIKEN cDNA 4921511D23 gene

Contactin 1

Transcrpition

Nsep1

Crx

Deaf1

Ddb1

Mov10l1

H2afz

Hoxc6

Polg

2810411G20Rik

9530043P15Rik

Cdr2

$\mathrm{Hmx} 3$

Nfkbib
Nuclease sensitive element binding protein 1

Cone-rod homeobox containing gene

Deformed epidermal autoregulatory factor 1 (Drosophila)

Damage specific DNA binding protein 1

Moloney leukemia virus 10-like 1

H2A histone family, member Z

Homeobox C6

Polymerase (DNA directed), gamma

RIKEN cDNA 3010019003 gene

Ribonuclease A family, member 6

Cerebellar degeneration-related 2

H6 homeobox 3

Nuclear factor, kappa light chain gene enhancer inhibitor, beta
1.45

1.42

1.17

4.27

1.30

1.26

1.45

1.35

1.56

1.48

1.38

1.30

1.40

1.23

1.36

0.60

1.45

0.70

1.67

1.28
$<0.010$

$$
<0.010
$$

$<0.010$

$<0.010$

$<0.010$

$<0.010$

$<0.010$

$<0.010$

$<0.010$

$<0.010$

$<0.010$

$<0.010$

$<0.010$

$<0.010$

$<0.010$

$<0.010$

$<0.010$

$<0.010$

$<0.010$

$<0.010$
1.16

1.31

1.06

1.51

0.99

1.10

1.18

1.01

1.06

1.27

1.08

1.19

1.21

1.02

1.16

0.63

1.24

0.94

1.16

1.16
$<0.010$

0.012

0.046

$<0.010$

NS

NS

NS

Intracellular trafficking

$\begin{array}{ll}\text { Syngr3 } & \text { Synaptogyrin 3 } \\ \text { Nup210 } & \text { Nucleoporin 210 }\end{array}$

Stx6 $\quad$ Syntaxin 6

Poll Polymerase (DNA directed), lambda

2700038C24Rik Exportin 5

Cytoskeletal proteins

Krt1-5

Tln

Cappb1

GTPase-associated

Gna-rs1

2600013G09Rik

Keratin complex 1 , acidic, gene 5

Talin

Capping protein (actin filament) muscle Z-line, beta

Guanine nucleotide-binding protein,

RAB, member of RAS oncogene family-like 4

Protein

Pfdn5

G1rzfp-pending

1600016E11Rik

2310004B22Rik

Dnajc5

4933406J07Rik 3300002I08Rik

AW494535

2610302I02Rik

Olfr67

1110019I12Rik

Sepm

Prefoldin 5/protein folding

Ring finger protein 130

Prolactin-like protein I/hormone activity

RIKEN cDNA 4930404J24 gene

Hsp40 homolog, subfamily C, member 5 molecular chaperone

RIKEN cDNA 4933406J07 gene/KRAB protein

RIKEN cDNA 3300002I08 gene/KRAB protein

F-box only protein 4

Purinergic receptor (family A group 5) G-protein

Olfactory receptor $67 / \mathrm{G}$-protein coupled receptor protein

Selenoprotein N, 1 unknown fn

Selenoprotein M

Ribosomal proteins

Rpl37a

Rpl27a

Mrpl53

Ribosomal protein L37a

Ribosomal protein L27a

Mitochondrial ribosomal protein L53

Rps5

Ribosomal protein S5
1.32

1.41

1.30

1.43

0.77

2.25

1.19

0.62

$<0.010$

$<0.010$

$<0.010$

$<0.010$

$<0.010$

$<0.010$

$<0.010$

$<0.010$

1.43

0.50

$<0.01$

$<0.01$

0.45

1.54

1.47

1.42

1.23

1.30

0.62

1.27

1.23

1.39

1.49

1.28

1.30

1.11

1.12

0.97

1.29

1.12

0.71

1.21

0.86

$<0.010$

$<0.010$

$<0.010$

$<0.010$

$<0.010$

$<0.010$

$<0.010$

0.000

$<0.010$

$<0.010$

$<0.010$

$<0.010$

1.50

1.41

1.62

1.24

0.51

1.97

0.49
$<0.010$

$<0.010$

$<0.010$

$<0.010$

$<0.010$

$<0.010$

$<0.010$
0.62

1.03

1.19

1.33

1.09

1.02

0.93

1.28

1.11

1.25

1.17

1.31

1.37

1.18

1.22

1.17

NS

NS

0.021

NS

0.028

0.012

NS

0.012

0.016

NS

NS

NS

0.036

$<0.010$

$<0.010$

NS

NS

NS

0.021

$<0.010$

0.016

$<0.010$

NS 
Table 2 Continued

\begin{tabular}{|c|c|c|c|c|c|}
\hline Gene & Gene description & $\begin{array}{c}B 6: D C \\
(12 \text { weeks })\end{array}$ & $\mathrm{P}$-value & $\begin{array}{c}B 6: D C \\
(8 \text { weeks })\end{array}$ & $\mathrm{P}$-value \\
\hline \multicolumn{6}{|l|}{ Proteinkinase } \\
\hline Gsk3b & Glycogen synthase kinase 3 beta & 1.43 & $<0.010$ & 1.08 & NS \\
\hline Dyrk1a & $\begin{array}{l}\text { Dual-specificity tyrosine-(Y)-phosphorylation regulated } \\
\text { kinase 1a }\end{array}$ & 1.31 & $<0.010$ & 1.24 & $<0.010$ \\
\hline Ask-pending & Expressed sequence AA545217 & 0.66 & $<0.010$ & 0.96 & NS \\
\hline Map2k4 & Mitogen activated protein kinase kinase 4 & 1.23 & $<0.010$ & 1.18 & 0.012 \\
\hline Srpk2 & Serine/arginine-rich protein specific kinase 2 & 1.39 & $<0.010$ & 1.17 & NS \\
\hline ACO & Human-kallikrein 15 & 0.52 & $<0.010$ & 1.01 & NS \\
\hline 0610025G21Rik & RIKEN cDNA 0610025G21 gene & 0.68 & $<0.010$ & 0.89 & NS \\
\hline 6330415M09Rik & RIKEN cDNA 63300415M09 gene & 0.75 & $<0.010$ & 0.86 & NS \\
\hline \multicolumn{6}{|l|}{ Membrane protein } \\
\hline Fut8 & Fucosyltransferase 8 & 0.61 & $<0.010$ & 0.94 & NS \\
\hline $\mathrm{B} 2 \mathrm{~m}$ & Beta-2 microglobulin & 1.97 & $<0.010$ & 1.06 & NS \\
\hline Hs3st3b & Heparan sulfate (glucosamine) 3-O-sulfotransferase 3B & 3.14 & $<0.010$ & 1.48 & $<0.010$ \\
\hline Jcam2 & Junction adhesion molecule 2 & 1.67 & $<0.010$ & 1.42 & 0.016 \\
\hline Gpc6 & Glypican 6 & 1.44 & $<0.010$ & 1.31 & 0.036 \\
\hline 1700013004Rik & Chemokine-like factor super family $2 \mathrm{~B}$ & 1.15 & $<0.010$ & 1.03 & NS \\
\hline 3110001D03Rik & RIKEN cDNA 3110001D03 gene & 1.18 & $<0.010$ & 1.16 & $<0.010$ \\
\hline \multicolumn{6}{|l|}{ Immunity } \\
\hline Mtcp1 & Mature T-cell proliferation 1 & 0.79 & $<0.010$ & 1.05 & NS \\
\hline Cd5 & CD5 antigen & 1.36 & $<0.010$ & 1.19 & 0.028 \\
\hline 2410003B16Rik & RIKEN cDNA 2410003B16 gene & 1.30 & $<0.010$ & 1.10 & NS \\
\hline 2310043N20Rik & Interleukin 1 family, member 8 & 0.44 & $<0.010$ & 0.58 & 0.028 \\
\hline \multicolumn{6}{|l|}{ Neurotoxin activity } \\
\hline Lynx1 & $\begin{array}{l}\text { Ly6/neurotoxin } 1 / \mathrm{Chrnb} 2 \text { cholinergic receptor, nicotinic, } \\
\text { beta polypeptide } 2\end{array}$ & 1.45 & $<0.010$ & 1.41 & $<0.010$ \\
\hline \multicolumn{6}{|l|}{ Lipid metabolism } \\
\hline Hsd17b4 & Hydroxysteroid (17-beta) dehydrogenase 4 & 1.38 & $<0.010$ & 1.17 & NS \\
\hline \multicolumn{6}{|c|}{ Carbohydrate metabolism } \\
\hline Glce & Glucuronyl C5-epimerase & 1.47 & $<0.010$ & 1.28 & $<0.010$ \\
\hline Uae1 & Glucosamine & 1.51 & $<0.010$ & 1.27 & $<0.010$ \\
\hline \multicolumn{6}{|l|}{ Extracellular matrix } \\
\hline Dspp & Dentin sialophosphoprotein & 1.35 & $<0.010$ & 1.11 & NS \\
\hline \multicolumn{6}{|c|}{ Inhibits the growth of E. coli } \\
\hline AB030186 & RIKEN cDNA B230317C12 gene & 1.40 & $<0.010$ & 1.25 & 0.016 \\
\hline \multicolumn{6}{|l|}{ Ion channel } \\
\hline Cdh7 & Cadherin 7 , type 2 & 1.29 & $<0.010$ & 1.17 & 0.047 \\
\hline Atp8a1 & $\begin{array}{l}\text { ATPase, aminophospholipid transporter (APLT), class I, } \\
\text { type 8A, member } 1\end{array}$ & 1.41 & $<0.010$ & 1.06 & NS \\
\hline Aqp2 & Aquaporin 2 & 1.28 & $<0.010$ & 1.15 & NS \\
\hline \multicolumn{6}{|l|}{ Function not known } \\
\hline 1700080G18Rik & RIKEN cDNA $1700080 G 18$ gene & 0.58 & $<0.010$ & 0.77 & $<0.010$ \\
\hline 4931406E20Rik & RIKEN cDNA 4931406E20 gene & 0.54 & $<0.010$ & 0.63 & $<0.010$ \\
\hline 4933437I04Rik & RIKEN cDNA 4933437I04 gene & 1.70 & $<0.010$ & 1.30 & $<0.010$ \\
\hline 1110012O05Rik & RIKEN cDNA 1110012005 gene & 1.42 & $<0.010$ & 0.96 & NS \\
\hline 1700113H08Rik & RIKEN cDNA 1700113 H08 gene & 0.81 & $<0.010$ & 0.88 & NS \\
\hline 2210008F15Rik & Nucleolar protein 7 & 1.34 & $<0.010$ & 1.04 & NS \\
\hline $2900010 \mathrm{~m} 23$ rik & RIKEN cDNA 2900010M23 gene & 1.36 & $<0.010$ & 1.15 & NS \\
\hline 4930415G15Rik & RIKEN cDNA 4930415G15 gene & 0.70 & $<0.010$ & 0.94 & NS \\
\hline 1700042B14Rik & RIKEN cDNA 1700042B14 gene & 1.44 & $<0.010$ & 1.08 & NS \\
\hline D630002J15Rik & RIKEN cDNA D630002J15 gene & 1.34 & $<0.010$ & 1.24 & $<0.010$ \\
\hline 1190006A08Rik & RIKEN cDNA 1190006A08 gene & 1.94 & $<0.010$ & 1.20 & 0.047 \\
\hline 1110037No9Rik & RIKEN cDNA 1110037N09 gene & 0.76 & $<0.010$ & 0.88 & 0.016 \\
\hline 4933427L07Rik & RIKEN cDNA 4933427L07 gene & 1.33 & $<0.010$ & 1.07 & NS \\
\hline 1500032H18Rik & RIKEN cDNA $1500032 \mathrm{H} 18$ gene & 0.76 & $<0.010$ & 1.07 & NS \\
\hline 5730528L13Rik & RIKEN cDNA 5730528L13 gene & 0.61 & $<0.010$ & 1.00 & NS \\
\hline 1810019P04Rik & RIKEN cDNA 1810019P04 gene & 2.71 & $<0.010$ & 1.50 & 0.028 \\
\hline 2610036L13Rik & RIKEN cDNA 2610036L13 gene & 1.39 & $<0.010$ & 1.19 & 0.016 \\
\hline LOC207933 & Isopentenyl-diphosphate delta-isomerase-like & 0.52 & $<0.010$ & 0.72 & NS \\
\hline 4932702K14Rik & RIKEN cDNA 4932702K14 gene & 1.63 & $<0.010$ & 1.59 & NS \\
\hline
\end{tabular}


Table 2 Continued

\begin{tabular}{|c|c|c|c|c|c|}
\hline Gene & Gene description & $\begin{array}{c}B 6: D C \\
(12 \text { weeks) }\end{array}$ & P-value & $\begin{array}{c}B 6: D C \\
(8 \text { weeks })\end{array}$ & $\mathrm{P}$-value \\
\hline 0610011F06Rik & RIKEN cDNA 0610011F06 gene & 2.21 & $<0.010$ & 1.23 & 0.028 \\
\hline 1700013E09Rik & RIKEN cDNA 1700013E09 gene & 1.44 & $<0.010$ & 1.32 & $<0.010$ \\
\hline 4921517J23Rik & RIKEN cDNA 4921517J23 gene & 0.75 & $<0.010$ & 1.01 & NS \\
\hline 3110001E11Rik & Unknown fn & 1.26 & $<0.010$ & 1.22 & $<0.010$ \\
\hline C79747 & Unknown (protein for image:3491533) & 1.92 & $<0.010$ & 1.13 & NS \\
\hline 1700012B18Rik & Pregnancy-induced growth inhibitor & 10.15 & $<0.010$ & 2.70 & NS \\
\hline
\end{tabular}

Table 3 Verification of selected genes by semiquantitative RT-PCR

\begin{tabular}{|c|c|c|c|c|c|c|}
\hline & 8 weeks & $M A-8$ weeks & SQ-8 weeks & 12 weeks & MA-12 weeks & SQ-12 weeks \\
\hline 1 & 1700012B18Rik & $2.70^{\mathrm{a}}$ & 2.31 & B18Rik & 10.15 & 2.52 \\
\hline 2 & Traf6 & 2.67 & 1.78 & $P d e 4 b$ & 4.27 & 2.18 \\
\hline 3 & 1700112L09Rik & 2.16 & 1.91 & BaffR & 1.30 & 1.41 \\
\hline 4 & Pde $4 b$ & 1.51 & 1.41 & Stat1 & 1.33 & 1.48 \\
\hline 5 & Stat1 & 1.20 & 1.16 & Map2k4 & 1.18 & 1.22 \\
\hline 6 & Map2k4 & 1.18 & 1.09 & Rac 1 & 1.18 & 1.18 \\
\hline 7 & Baff & 1.10 & 1.11 & p38beta & 1.07 & 1.35 \\
\hline 8 & Rac 1 & 1.06 & 1.06 & Baff & 0.97 & 0.96 \\
\hline 9 & BaffR & 0.96 & 1.06 & Traf6 & 0.95 & 0.96 \\
\hline 10 & Jnk & 0.93 & 1.07 & Jnk & 1.05 & 1.05 \\
\hline 11 & p38beta & 0.82 & 0.77 & $N f k b$ & 1.04 & 1.04 \\
\hline 12 & Adh5 & 0.66 & 0.78 & Pfdn5 & 0.45 & 0.64 \\
\hline 13 & Pfdn5 & 0.62 & 0.71 & Adh5 & 0.49 & 0.55 \\
\hline 14 & $N f k b$ & 0.59 & 0.79 & 1700112Lo9Rik & 0.40 & 0.46 \\
\hline
\end{tabular}

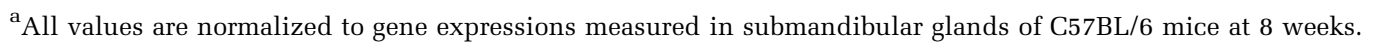

mice revealed 90 genes that reached statistical significance. (These data can be found in Supplemental Figure 1 and Supplemental Table 1.) Many of these genes appear to be involved in neural or epithelial cell development, as well as cellular homeostasis. All proved to be distinct from the genes differentially expressed in 12-week-old C57BL/6.NOD-Aec1Aec2 submandibular glands.

\section{Validation of Microarray Data by SemiQuantitative RT-PCR}

To verify the overall results obtained from the microarrays, a number of genes were selected randomly for semiquantitative RT-PCR analysis. Genes were selected that were expressed at higher levels, lower levels and equal levels between the C57BL/6.NOD-Aec1Aec2 and C57BL/6 mice, as determined by microarray analyses. As shown in Table 3, the relative expression of these genes in the submandibular glands, as determined by RT-PCR, proved highly consistent with the relative expressions obtained from the microarrays. Furthermore, the calculated differential expressions proved to be greater by microarray than by RT-PCR, consistent with published data (Eckenrode et $a l^{25}$ and personal observations).

\section{Association between Selected Differentially-Expressed Genes and SjS-like Autoimmune Exocrinopathy}

Identification of large numbers of individual genes that are differentially expressed temporally can present a daunting task in data interpretation. However, this task can be simplified if several functionally associated genes can be linked. One interesting example of functionally linked genes identified by microarray involves cellular apoptosis. As reported in our earlier studies, ${ }^{4,7,9,26}$ a wave of apoptosis occurs in the early phases of SjS-like disease, during which time there is a concomitant increased expression of caspase 3. As presented in Table 4, the caspase 3 gene was found to be highly upregulated at 8 weeks of age in the submandibular glands of C57BL/6.NOD-Aec1Aec2 mice, when analyzed by the Student's $t$ test. Unexpectedly, however, a second caspase gene, caspase 11, was also significantly upregulated, while other caspase genes and mitochondria-associated apoptotic genes were not. As caspase-11 is generally induced by proinflammatory stimuli (Schauvliege et $a 2^{27}$ especially IL-1 and tumor necrosis factor (TNF)-associated proteins, genes of the TNF superfamily represented on the array were selected and examined. As listed in Table 5 in the order of differential expression observed at 8 weeks of age, only about 
Table 4 Expression of apoptosis-related genes

\begin{tabular}{|c|c|c|c|c|c|}
\hline Gene & Full name & 8 weeks & $\mathrm{P}$-values $\left(8\right.$ weeks) ${ }^{\mathrm{a}}$ & 12 weeks & $\mathrm{P}$-values $\left(12\right.$ weeks $^{\mathrm{a}}$ \\
\hline Casp2 & Caspase 2 & 1.25 & 0.02 & 1.19 & 0.14 \\
\hline Casp3 & Caspase 3 & 2.17 & 0.47 & 0.92 & 0.68 \\
\hline Casp8ap2 & Caspase 8 -associated protein 2 & 0.81 & 0.05 & 0.97 & 0.80 \\
\hline Cflar & Casp8 and fadd-like apoptosis regulator & 0.94 & 0.47 & 0.94 & 0.28 \\
\hline Casp9 & Caspase 9 & 0.71 & 0.03 & 0.67 & 0.01 \\
\hline Casp11 & Caspase 11 & 2.60 & 0.39 & 0.95 & 0.69 \\
\hline Casp12 & Caspase 12 & 0.90 & 0.23 & 1.09 & 0.22 \\
\hline Casp14 & Caspase 14 & 0.89 & 0.39 & 0.88 & 0.01 \\
\hline Bad & Bcl2-associated death promoter & 0.91 & 0.62 & 1.13 & 0.30 \\
\hline Bak1 & Bcl2 antagonist/killer & 0.61 & 0.03 & 0.53 & 0.04 \\
\hline $\operatorname{Bax}$ & Bcl2-associated X protein & 0.92 & 0.64 & 0.83 & 0.15 \\
\hline bag1 & Bcl2-associated athanogene 1 & 1.06 & 0.67 & 1.19 & 0.27 \\
\hline $\operatorname{daxx}$ & Fas death domain-associated protein & 0.86 & 0.29 & 1.19 & 0.28 \\
\hline
\end{tabular}

${ }^{\text {a }} P$-values determined by the Student's $t$ test.

Table 5 Differentially expressed tumor necrosis factor (TNF) super family genes at 8 and 12 weeks of age as determined by microarray analyses

\begin{tabular}{|c|c|c|c|c|c|}
\hline Gene & Name of gene & $8 w_{e e k s}{ }^{\mathrm{a}}$ & P-values $(8 \text { weeks })^{\mathrm{a}}$ & 12 weeks & P-values $(12 \text { weeks })^{\mathrm{a}}$ \\
\hline traf6 & TNF receptor-associated factor 6 & 2.67 & 0.06 & 0.95 & 0.180 \\
\hline tnfip6 & TNF-induced protein 6 & 2.65 & 0.04 & 0.97 & 0.204 \\
\hline tnfaip1 & TNF-induced protein 1 & 2.10 & 0.06 & 0.69 & 0.16 \\
\hline tnfrsf $13 b$ & TNF-receptor superfamily, member $13 \mathrm{~b}$ & 1.83 & 0.5 & 1.17 & 0.5 \\
\hline $\operatorname{tnf} s f 7$ & TNF (ligand) superfamily, member 7 & 1.38 & 0.056 & 1.11 & 0.04 \\
\hline traf1 & TNF-receptor-associated factor 1 & 1.31 & 0.26 & 1.09 & 0.834 \\
\hline litaf & Lps-induced TNF-alpha factor & 1.25 & 0.6 & 0.92 & 0.013 \\
\hline $\operatorname{tnf} f \mathrm{~s} g$ & TNF (ligand) superfamily, member 9 & 1.22 & 0.14 & 0.97 & 0.466 \\
\hline tnfaip3 & TNF-induced protein 3 & 1.13 & 0.42 & 1.35 & 0.04 \\
\hline $\operatorname{tnf} s f 13 b$ & TNF (ligand) superfamily, member $13 \mathrm{~b}$ & 1.10 & 0.103 & 0.97 & 0.51 \\
\hline $\operatorname{tnfsf13}$ & TNF (ligand) superfamily, member 13 & 1.10 & 0.916 & 0.97 & 0.34 \\
\hline $\operatorname{tnfsf8}$ & TNF (ligand) superfamily, member 8 & 1.07 & 0.012 & 0.69 & 0.06 \\
\hline tnfrsf19 & TNF-receptor superfamily, member 19 & 1.01 & 0.013 & 1.26 & 0.05 \\
\hline $\operatorname{tnfrsf8}$ & TNF-receptor superfamily, member 8 & 0.98 & 0.446 & 1.03 & 0.09 \\
\hline $\operatorname{tnf} s f 14$ & TNF (ligand) superfamily, member 14 & 0.98 & 0.287 & 1.19 & 0.06 \\
\hline $\operatorname{tnfsf4}$ & Tax-transcriptionally activated glycoprotein 1 ligand & 0.97 & 0.116 & 0.85 & 0.980 \\
\hline $\operatorname{tnfrsf13c}$ & Baff receptor & 0.96 & 0.013 & 1.30 & 0.06 \\
\hline ripk1 & Receptor (tnfrsf)-interacting serine-threonine kinase 1 & 0.92 & 0.06 & 0.92 & 0.790 \\
\hline tnfrsf21 & TNF-receptor superfamily, member 21 & 0.92 & 0.072 & 1.07 & 0.743 \\
\hline tnfrsf23 & Tumor necrosis factor receptor superfamily, member 23 & 0.90 & 0.879 & 0.95 & 0.05 \\
\hline tnfrsf12 & TNF-receptor superfamily, member 12 & 0.85 & 0.023 & 1.06 & 0.096 \\
\hline traf3 & TNF receptor-associated factor 3 & 0.84 & 0.04 & 1.25 & 0.0425 \\
\hline $\operatorname{tnfrsf5}$ & TNF-receptor superfamily, member 5 & 0.82 & 0.105 & 1.04 & 0.214 \\
\hline tnfrsf11a & TNF-receptor superfamily, member $11 \mathrm{a}$ & 0.81 & 0.88 & 0.84 & 0.126 \\
\hline tnfaip2 & TNF-induced protein 2 & 0.81 & 0.089 & 0.86 & 0.0421 \\
\hline traf4 & TNF-receptor associated factor 4 & 0.80 & 0.065 & 0.75 & 0.056 \\
\hline tnfrsf 4 & Tax-transcriptionally activated glycoprotein 1 & 0.79 & 0.045 & 0.92 & 0.013 \\
\hline tnfrsf17 & TNF-receptor superfamily, member 17 & 0.78 & 0.068 & 1.11 & 0.011 \\
\hline $\operatorname{tnf} s f 10$ & TNF-related apoptosis inducing ligand & 0.73 & 0.06 & 0.72 & 0.033 \\
\hline
\end{tabular}

${ }^{\mathrm{a}}$ Expression of TNF genes in submandibular glands of C57BL/6J.NOD-Aec1Aec2 mice are listed in order of expression at 8 weeks of age normalized against 8-week-old C57BL/6J mice.

six of the nearly $30 \mathrm{TNF}$ genes, that is, traf6, tnfip6, tnfaip1, tnfrsf13b, tnfsf7 and traf1, were upregulated when compared to those in sex- and age-matched C57BL/6 mice. While most of these genes showed a relative loss of upregulated expression by 12 weeks of age, tnfaip3 (TNF-alpha inducible protein-1), traf3, tnfrsf19 (TAJ/TROY), tnfrsf13 (BAFF receptor) and possibly tnfsf14 (LIGHT) were temporally upregulated in the submandibular glands at 12 weeks as compared to 8 weeks of age. Thus, these results are consistent with immunohistochemical data indicating that a wave of apoptosis occurs around 8 weeks of age in the submandibular glands of C57BL/6.NOD-Aec1Aec2 or NOD mice ${ }^{26,28}$ and, at the same time, provides evidence of a possible novel pathway involving caspase-11 underlying acinar cell apoptosis. Similarly, an upregulation of TAJ/TROY, BAFF-receptor and LIGHT at 12 weeks 


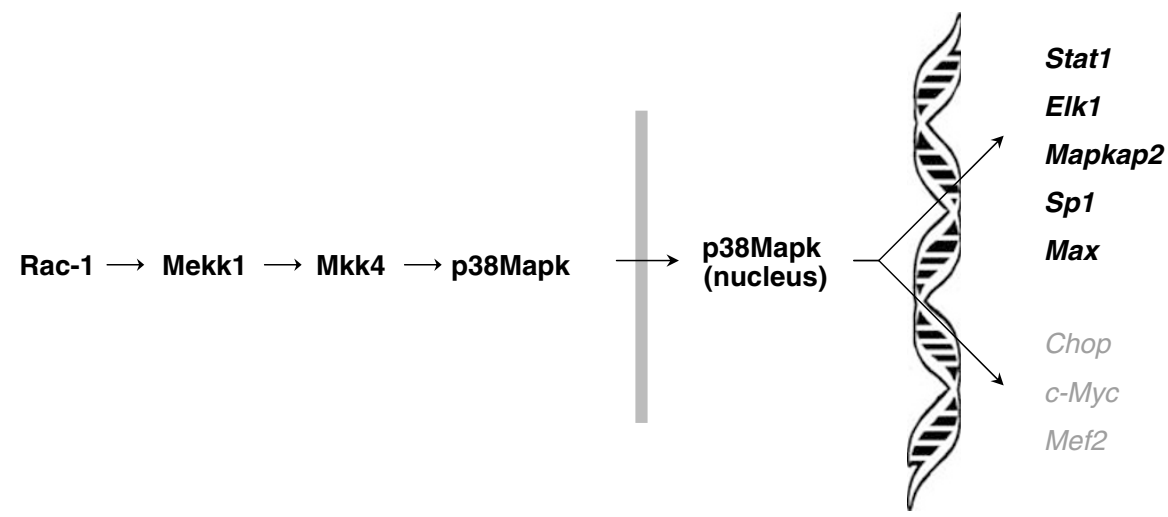

Figure 3 Differential regulation of genes in the submandibular glands of C57BL/6.NOD-Aec1Aec2 mice between 8 and 12 weeks of age controlled through the Rac-1 signal transduction pathway. Genes upregulated are indicated in bold, while those downregulated are indicated in grey. Genes within this set but not on the microarray include Atf2, Msk1, Creb, Histone H3 and Hmg14.

suggests an appearance of neuro-immunological processes, known to begin around this time.

A second set of functionally associated genes involving the Rac1/MEKK1 signal transduction pathway was examined based on the strong change in the relative gene expression levels exhibited by chimerin (Rac1 suppressor) between 8 weeks and 12 weeks of age (ie, a 2.16-fold increase vs a 2.5-fold decrease, respectively) (see Table 2). The Rac1/PI3 K and Rac1/MEKK1 signal transduction pathways are involved in cell proliferation, survival and programmed cell death involving the PKA, ERK1/2, SAPK/JNK and p38-MAPK downstream pathways. Genes within these various pathways present on the array were found to be upregulated significantly in the submandibular glands at 12 weeks of age compared to 8 weeks of age (Figure 3), as determined by the Student's $t$ test, and these differential expressions were confirmed by RT-PCR profiling. When analyzed more closely, other genes, especially nuclear-associated genes such as c-jun, elk1, sp1, max, Mapkap2 and stat1, were upregulated, while the genes chop, met2 and $c-m y c$ were found to be downregulated. Interestingly, the upregulation of $N F-k B$ was coordinated with the upregulated expression of mekk1. Thus, these microarray data point to major temporal changes in gene expression patterns indicative of major alterations in the homeostasis of the submandibular glands between 8 and 12 weeks of age.

\section{Discussion}

With the present study, we have applied microarray technology to identify genes that are differentially regulated during early development and onset of SjS-like disease associated with the NOD mouse model. To reduce background noise, we have taken advantage of our recently constructed C57BL/ 6.NOD-Aec1Aec2 mouse strain, a C57BL/6J mouse containing two genetic regions derived from the NOD mouse capable of conferring full-blown SjS- like disease in the resulting congenic mice. ${ }^{23}$ This permits a direct comparison between disease-prone C57BL/6.NOD-Aec1Aec2 mice and their parental C57BL/6 partners that exhibit no evidence of SjSlike disease at the time points evaluated. Previous investigations of C57BL/6.NOD-Aec1Aec2 mice have shown that the $A e c 2$ region on chromosome 1 regulates the numerous pre-disease pathophysiological changes in the salivary and lacrimal glands, while the Aec1 region on chromosome 3 controls the autoimmune response leading to clinical disease. ${ }^{2,23}$ Thus, this C57BL/6J-C57BL/6.NOD-Aec1Aec2 congenic mouse combination represents an excellent model for identifying candidate genes responsible for both the early development and eventual onset of SjS-like disease.

To identify candidate genes possibly involved in the early phases of autoimmune xerostomia, we carried out microarray analyses using cDNA prepared from submandibular glands of C57BL/6.NODAec1Aec2 and C57BL/6 mice at two ages: 8 weeks, when the mice exhibit an early preclinical disease, and 12 weeks, when the mice exhibit the first overt signs of an impending autoimmune disease. Histological examinations of the submandibular glands show increased acinar cell apoptosis ${ }^{13,28}$ at the 8 week time point, and first signs of leukocytic infiltration at the 12 week time point,, ${ }^{2,23}$ although the presence of small numbers of leukocytes (especially dendritic cells and macrophages) are no doubt present in the submandibular glands at both time points. Our comparison of gene expressions between C57BL/6J and C57BL/6.NOD-Aec1Aec2 mice identified some 75 genes at 8 weeks and 105 genes at 12 weeks of age that were differentially expressed $(P<0.01)$ based on the Mann-Whitney $U$ test, with additional genes being identified using less stringent criteria with the Student's $t$ test. While genes differentially expressed at 8 weeks of age in the C57BL/6.NOD-Aec1Aec2 mouse encode products primarily involved in normal cellular processes like transcription, translation, DNA replication, signal transduction, vesicle trafficking and 
metabolism, the genes differentially expressed at 12 weeks of age encode additional products involved in oxidative phosphorylation, free-radical neutralization, ion channel activity, protein processing and immune function. Using semi-quantitative RT-PCR, we were able to validate the relative levels of gene expressions observed with microarrays. Overall, these data indicate major changes in cellular homeostasis occur in the submandibular glands of C57BL/ 6.NOD-Aec1Aec2 mice during this critical 4 week time frame.

Based on genes differentially expressed at a statistically significant level, together with genes that are known to cluster with the identified genes, a number of signal transduction pathways could be identified that apparently are temporally activated and potentially important to onset of SjS-like disease. Several of these pathways are depicted in Figures 4 and 5, and discussed in detail below. Genes differentially expressed in the submandibular glands of C57BL/6.NOD-Aec1Aec2 mice at 8 weeks of age compared to those of age- and sex-matched C57BL/6J mice include several genes encoding molecules associated with apoptosis, for example, F8a, Aplp2, Capn10, Cebpa and Xrcc5 (Ku80). In addition, two cysteine protease genes known to enhance apoptosis, specifically those encoding for caspase-3 and caspase-11, are both upregulated at 8 weeks. While caspase-11 is not expressed constitutively in most cell types, its expression is rapidly induced by proinflammatory stimuli such as LPS or IFN- $\gamma$ as a result of NF-kB and/or STAT1 binding to its promoter. $^{27}$ While activation of caspase- 11 can lead to caspase-3 activation, it can also promote caspase-1 activation and subsequent production of IL- $1 \beta$ and/or IL- $18 .^{29}$ Based on the fact that $N F-k B$ is not notably upregulated until 12 weeks of age together with our earlier observation in the NOD mouse that expression of IFN- $\gamma$ in the submandibular gland is increased in very young mice, ${ }^{30}$ we would propose that caspase-11 expression at 8 weeks of age is through STAT1 rather than NF-kB (see Figure 4).

This upregulation of the caspase-11 and caspase3 genes rather than caspase- 9 and bax, together with alterations in specific TNF/TNFR family gene expressions (especially traf6 and traf1) and the early presence of IFN- $\gamma$, all support the hypothesis that an increase in proinflammatory cytokines (possibly from pattern recognition of extrinsic bacterial/viral

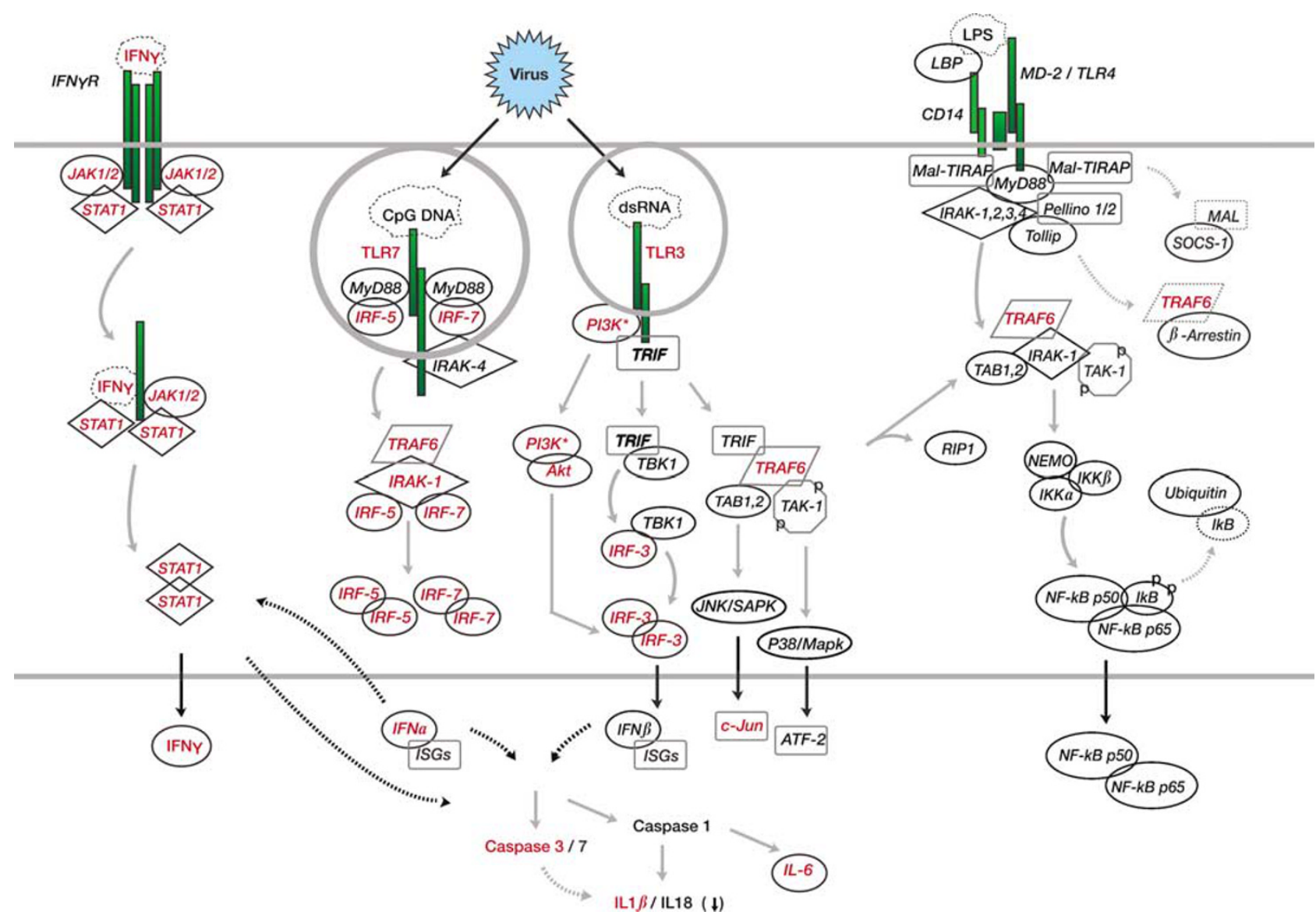

Figure 4 Summary of upregulated genes and signal transduction pathways in submandibular glands of C57BL/6.NOD-Aec1Aec2 mice at 8 weeks of age. Genes found to be upregulated are shown in red, while genes found to be downregulated or not represented on the microarrays are shown in black. Discussion of these genes and pathways is presented in the text. 


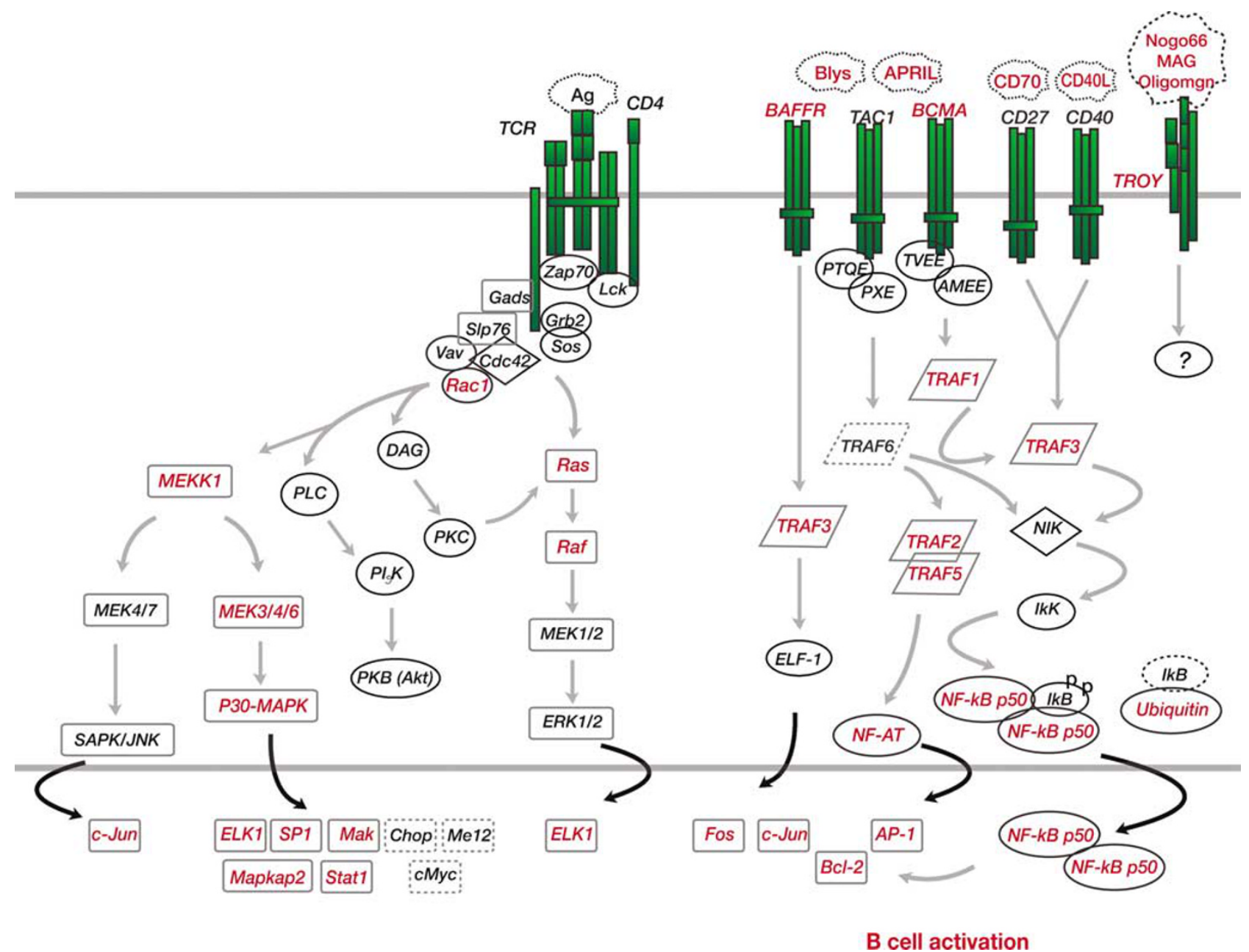

Figure 5 Summary of upregulated genes and signal transduction pathways in submandibular glands of C57BL/6.NOD-Aec1Aec2 mice at 12 weeks of age. Genes found to be upregulated are shown in red, while genes found to be downregulated or not represented on the microarrays are shown in black. Discussion of these genes and pathways is presented in the text.

pathogen stimuli through toll-like receptor (TLR) signal transduction pathways) leads to the activation of cell death-mediated or ER-mediated rather than mitochondria-mediated pathways. Consistent with this concept is the known increased expressions of ICE, NEDD-2 and Apopain/CCP in NOD mice. ${ }^{28}$ Interestingly, the TLR pathways whose genes were identified as being upregulated involves Irf3, Irf5 and Irf7 signaling, all endosome-associated TLRs (Figure 4). Genes associated with TLR pathways whose recognition patterns generally involve bacteria (TLR4, TLR1, TLR2 and TLR6) remained unchanged. Nevertheless, it remains premature to conclude that specific cell death-mediated and/or ER-mediated pathways are responsible for the enhanced apoptosis in the exocrine glands of C57BL/6.NOD-Aec1Aec2 mice prior to disease onset without first examining more closely, by other experimental approaches, molecules that are involved in mitochondria-mediated pathways, for example, cytochrome $c$, Apaf-1, Bax and caspase-9. In any event, since apoptosis and other intrinsic alterations appear to play a major role in the early stages of SjS-like disease in the mouse model, and possibly in humans, blocking the early apoptotic events by targeting caspase-11 (or its human ortholog, caspase-5) represents a new possibility.

Of those genes exhibiting the highest statistical significant differential expressions in the submandibular glands of C57BL/6.NOD-Aec1Aec2 mice at 8 weeks of age, which include $F 8 a$ (factor VIIIassociated gene A located within intron 22 and associated with Haemophilia A) ${ }^{31}$ and Cox-6a (terminal enzyme of the mitochondrial electron transport chain that catalyzes the transfer of electrons from cytochrome $c$ to oxygen and directly related to energy demand through neural activity or muscle adaptation/hormonal changes), ${ }^{32}$ perhaps the more fascinating is 1700112LO9Rik encoding chimerin. Chimerin is a Rac-1 repressor that, despite being overexpressed more than two-fold in the submandibular glands at 8 weeks, is strongly downregulated at 12 weeks of age, showing a direct relationship with an upregulation 
in Rac-1 expression. Rac1 is involved in signaling along several pathways, one involving PI3 $\mathrm{K}^{33,34}$ and PKB (Akt) ${ }^{35}$ and another involving MEKK1. ${ }^{36}$ Both cascades are important regulators of a wide spectrum of biological processes, including cell proliferation, differentiation, apoptosis, lipid metabolism and coagulation. Not surprising, then, genes within these pathways, like Mekk1, Mkk4, Map2k4, p38Mapk, Stat1 and Elk-1 also showed an upregulated expression, even though not always reaching statistical significance. In addition, Rac1 is known to be important in cytoskeletal changes involving E-cadherin and catenin p120/Vav $2^{37}$ possibly indicative of glandular remodeling. Since these pathways can be regulated by factors of the TNF super family of genes, a serious question to be asked is whether any link between upregulation of Rac-1 and any TNF-family proteins or receptors exists. This would once again focus attention on molecules encoded by traf6, tnfip6 and tnfaip1 that were upregulated at 8 weeks and downregulated at 12 weeks vs traf3, tnfaip3, tnfrsf13c, tnfsf14 and tnfrsf19 that were subsequently upregulated at 12 weeks. Also of interest is the reduced expression of traf1 that occurs between 8 and 12 weeks of age (Figures 4 and 5). A loss of Traf1 levels would permit higher expression levels of NIP45, a transcription factor that potentiates NF-kB-driven expression of IL-4, ${ }^{38}$ possibly explaining in part the important role IL-4 has been shown to play in development of SjS-like disease in NOD mice using IL-4 KO mice. ${ }^{3,39}$

One temporal inverse relationship worth investigating is that of traf3 vs traf6 gene expression in the submandibular glands of C57BL/6.NOD-Aec1Aec2 mice. As pointed out above, traf6 gene expression is upregulated at 8 weeks, but downregulated at 12 weeks, whereas traf3 gene expression is downregulated at 8 weeks and upregulated at 12 weeks. In a recent study, Häcker et $a l^{40}$ provided evidence that these two TRAF molecules are involved in controlling diverse signaling pathways. For example, TRAF3 is essential for the induction of type 1 IFNs and the cytokine IL-10, most likely through the recruitment of TBK-1/NAK. Interestingly, our microarray data indicate that both IL10ra and IL10rb genes are upregulated at 12 weeks of age. In addition, TRAF3 appears to be an important downstream transduction signal for BAFF-BAFFR, ${ }^{41}$ APRILBCMA, ${ }^{42}$ CD70-CD27, ${ }^{43}$ CD40L-CD40 ${ }^{41}$ and LIGHTHVEM, ${ }^{44}$ five systems thought to play a role in B lymphocyte hyper-reactivity of $\mathrm{SjS}$. As early signs of an overt autoimmune attack against the submandibular glands of C57BL/6.NOD-Aec1Aec2 mice can be observed by 12 weeks of age, including the appearance of leukocytes, it is important to note that three genes of the TNF superfamily, tnfrsf19 (TAJ/TROY), tnfrsf13 (BAFF receptor) and tnfsf14 (LIGHT) were upregulated in the submandibular glands at 12 weeks as compared to 8 weeks of age, suggesting active signaling processes occurring within infiltrating immune cells, the salivary gland epithelial and/ or neural cells per se (Figure 5). BAFF, for example, is known to regulate lymphocyte survival and activation. ${ }^{45}$ BAFF binds to three receptors, BAFF receptor (BAFF-R), transmembrane activator and cytophilin ligand interactor (TACI), as well as Bcell maturation antigen (BCMA) known to be closely associated with the development of germinal-like centers in the salivary glands of SjS patients. ${ }^{46,47}$ An upregulation of Nik, IKK and Nf-kB downstream of TACI and BCMA or Ppard, Cntn1, Poll and Dyrk1 associated with proliferation may account for the increased maturation and prolonged survival of $\mathrm{B}$ cells observed in both $\mathrm{SjS}$ patients and C57BL/ 6.NOD-Aec1Aec2 mice. Interestingly, a recent microarray analysis on minor salivary glands derived from human $\mathrm{SjS}$ patients also found upregulated BCMA (Tnfrsf17) ${ }^{48}$ consistent with our data from the mouse model. Similarly, LIGHT, which binds to the TR2 receptor of CD68-positive macrophages induces the phosphorylation of $\mathrm{I} k \mathrm{~B}$ and nuclear translocation of NF-kB. ${ }^{49,50}$ One potential consequence of LIGHT signaling, as observed in rheumatoid arthritis, ${ }^{50}$ might be the upregulation of matrix metalloprotease (MMP)-9 expression, and MMP9 has been shown to be actively induced in the submandibular glands of NOD mice during the disease state..$^{6,51}$ Lastly, an upregulation of tnfrsf19 (Taj/TROY) focuses attention on neural tissue involvement, and studies suggest that the parasympathetic neural system along with the muscarinic acetylcholine type-3 receptor are targets of the autoimmune process. ${ }^{16}$ Overall, then, while genes that are upregulated within the submandibular glands of C57BL/6.NOD-Aec1Aec2 mice of 8 weeks of age tend to point to apoptotic events, those upregulated at 12 weeks of age point strongly to early immunoregulatory events.

Other genes that are highly expressed in the submandibular glands of C57BL/6.NOD-Aec1Aec2 mice at 12 weeks of age include Pde $4 b$ and Krt1. Stimulation of TLR is known to upregulate Pde $4 b$ and production of PDE which, in turn, regulates intracellular levels of cAMP. Elevated intracellular cAMP has been associated with functional inhibition of numerous cell types such as lymphocytes, monocytes, macrophages, neutrophil, eosinophils, and mast cells. ${ }^{52}$ Inhibitors of PDE4 have shown the ability to suppress the in vitro responses of cells involved in the inflammatory process; ${ }^{52}$ however, only ablation of PDE4B impacted LPS signaling and TNF- $\alpha$ production, thereby demonstrating the highly specialized function of PDE4B in macrophages and its critical role in LPS signaling. ${ }^{53}$ Thus, the upregulated expression of Pde $4 b$ by 12 weeks of age may indicate the appearance of functionally impaired macrophages, often suggested for the NOD mouse, ${ }^{54}$ in the submandibular glands. Similarly, overexpression of Krt1 (keratin) may be a compensatory process for the loss of acinar cells and may explain excessive keratosis observed in the cornea during the disease state. 
In summary, these studies represent our initial attempt at using microarray technology to identify genes and gene clusters that may prove important in identifying underlying molecular mechanisms responsible for development of the physiological abnormalities, as well as regulating the autoimmune response, observed in the submandibular glands of C57BL/6.NOD.Aec1Aec2 mice prior to onset of the clinical disease. Although these studies cover only about one-third of the mouse genome, we have identified to date both distinct signal transduction pathways and individual genes that, based on previous studies of the pathophysiological changes occurring in the submandibular glands, represent excellent candidates regulating development of $\mathrm{SjS}$ like disease. These pathways (presented in Figures 3-5) suggest, when extrapolated, that there is an innate immunity that initiates an adaptive immunity favoring B lymphocyte activation. As we continue to mine the microarray data, it is now imperative to determine if such candidate genes and pathways are important or just our biased interpretation.

\section{Acknowledgements}

We thank Mr Jin Wang, Ms Grace Kim and Mr Eric Singson for their expert technical assistance. Supported in part by: PHS Grants DE014344 (ABP), DE015152 (ABP), DE55304 (ABP), U24 DE016509 (SC) from the NIH and the Sjögren's Syndrome Foundation (SC).

\section{References}

1 Jonsson R, Dowman SJ, Gordon T. Sjögren's syndrome. In: Koopman W (ed). Arthritis and Allied ConditionsA Textbook in Rheumatology, 15th edn. Lippincott Williams \& Wilkins: Philadelphia, 2004, pp 16811705.

2 Brayer J, Lowry J, Cha S, et al. Alleles from chromosomes 1 and 3 of NOD mice combine to influence Sjogren's syndrome-like autoimmune exocrinopathy. J Rheumatol 2000;27:1896-1904.

3 Brayer JB, Cha S, Nagashima H, et al. IL-4-dependent effector phase in autoimmune exocrinopathy as defined by the NOD.IL-4-gene knockout mouse model of Sjogren's syndrome. Scand J Immunol 2001;54: 133-140.

4 Cha S, Peck AB, Humphreys-Beher MG. Progress in understanding autoimmune exocrinopathy using the non-obese diabetic mouse: an update. Crit Rev Oral Biol Med 2002;13:5-16.

5 Robinson CP, Brayer J, Yamachika S, et al. Transfer of human serum IgG to nonobese diabetic Igmu null mice reveals a role for autoantibodies in the loss of secretory function of exocrine tissues in Sjogren's syndrome. Proc Natl Acad Sci USA 1998;95:7538-7543.

6 Yamachika S, Nanni JM, Nguyen KH, et al. Excessive synthesis of matrix metalloproteinases in exocrine tissues of NOD mouse models for Sjogren's syndrome. J Rheumatol 1998;25:2371-2380.
7 Humphreys-Beher MG, Peck AB. New concepts for the development of autoimmune exocrinopathy derived from studies with the NOD mouse model. Arch Oral Biol 1999;44(Suppl 1):S21-S25.

8 Cha S, Singson E, Cornelius J, et al. Muscarinic acetylcholine type-3 receptor desensitization due to chronic exposure to Sjogren's syndrome-associated autoantibodies. J Rheumatol 2006;33:296-306.

9 Humphreys-Beher MG, Hu Y, Nakagawa Y, et al. Utilization of the non-obese diabetic (NOD) mouse as an animal model for the study of secondary Sjogren's syndrome. Adv Exp Med Biol 1994;350:631-636.

10 Robinson CP, Yamachika S, Bounous DI, et al. A novel NOD-derived murine model of primary Sjogren's syndrome. Arthritis Rheum 1998;41:150-156.

11 Ishimaru N, Yoneda $\mathrm{T}$, Saegusa K, et al. Severe destructive autoimmune lesions with aging in murine Sjogren's syndrome through Fas-mediated apoptosis. Am J Pathol 2000;156:1557-1564.

12 Groom J, Kalled SL, Cutler AH, et al. Association of BAFF/BLyS overexpression and altered B cell differentiation with Sjogren's syndrome. J Clin Invest 2002; 109:59-68.

13 Robinson CP, Yamamoto H, Peck AB, et al. Genetically programmed development of salivary gland abnormalities in the NOD (nonobese diabetic)-scid mouse in the absence of detectable lymphocytic infiltration: a potential trigger for sialoadenitis of NOD mice. Clin Immunol Immunopathol 1996;79:50-59.

14 Ambrosetti A, Zanotti R, Pattaro C, et al. Most cases of primary salivary mucosa-associated lymphoid tissue lymphoma are associated either with Sjoegren syndrome or hepatitis $C$ virus infection. Triple association between hepatitis $\mathrm{C}$ virus infection, systemic autoimmune diseases, and B cell lymphoma. Br J Haematol 2004;126:43-49.

15 Cha S, van Blockland SC, Versnel MA, et al. Abnormal organogenesis in salivary gland development may initiate adult onset of autoimmune exocrinopathy. Exp Clin Immunogenet 2001;18:143-160.

16 Nguyen KH, Brayer J, Cha S, et al. Evidence for antimuscarinic acetylcholine receptor antibodymediated secretory dysfunction in nod mice. Arthritis Rheum 2000;43:2297-2306.

17 Gao J, Cha S, Jonsson R, et al. Detection of anti-type 3 muscarinic acetylcholine receptor autoantibodies in the sera of Sjogren's syndrome patients by use of a transfected cell line assay. Arthritis Rheum 2004;50: 2615-2621.

18 Cavill D, Waterman SA, Gordon TP. Antibodies raised against the second extracellular loop of the human muscarinic M3 receptor mimic functional autoantibodies in Sjogren's syndrome. Scand J Immunol 2004;59:261-266.

19 Dawson LJ, Field EA, Harmer AR, et al. Acetylcholineevoked calcium mobilization and ion channel activation in human labial gland acinar cells from patients with primary Sjogren's syndrome. Clin Exp Immunol 2001;124:480-485.

$20 \mathrm{Li} \mathrm{J}, \mathrm{Ha}$ YM, Ku NY, et al. Inhibitory effects of autoantibodies on the muscarinic receptors in Sjogren's syndrome. Lab Invest 2004;27:27.

21 Yui MA, Muralidharan K, Moreno-Altamirano B, et al. Production of congenic mouse strains carrying NODderived diabetogenic genetic intervals: an approach for the genetic dissection of complex traits. Mamm Genome 1996;7:331-334. 
22 Chesnut K, She JX, Cheng I, et al. Characterizations of candidate genes for IDD susceptibility from the diabetes-prone NOD mouse strain. Mamm Genome 1993;4:549-554.

23 Cha S, Nagashima H, Brown VB, et al. Two NOD Iddassociated intervals contribute synergistically to the development of autoimmune exocrinopathy (Sjogren's syndrome) on a healthy murine background. Arthritis Rheum 2002;46:1390-1398.

24 Takeichi M. Cadherin cell adhesion receptors as a morphogenetic regulator. Science 1991;251:14511455.

25 Eckenrode SE, Ruan Q, Yang P, et al. Gene expression profiles define a key checkpoint for type 1 diabetes in NOD mice. Diabetes 2004;53:366-375.

26 Humphreys-Beher MG, Peck AB, Dang H, et al. The role of apoptosis in the initiation of the autoimmune response in Sjogren's syndrome. Clin Exp Immunol 1999;116:383-387.

27 Schauvliege R, Vanrobaeys J, Schotte P, et al. Caspase11 gene expression in response to lipopolysaccharide and interferon-gamma requires nuclear factor-kappa B and signal transducer and activator of transcription (STAT) 1. J Biol Chem 2002;277:41624-41630 (E-pub 42002 Aug 41626).

28 Robinson CP, Yamachika S, Alford CE, et al. Elevated levels of cysteine protease activity in saliva and salivary glands of the nonobese diabetic (NOD) mouse model for Sjogren syndrome. Proc Natl Acad Sci USA 1997;94:5767-5771.

$29 \mathrm{Gu} \mathrm{Y}$, Kuida K, Tsutsui $\mathrm{H}$, et al. Activation of interferon-gamma inducing factor mediated by interleukin-1beta converting enzyme. Science 1997;275: 206-209.

30 Cha S, Brayer J, Gao J, et al. A dual role for interferon-gamma in the pathogenesis of Sjogren's syndrome-like autoimmune exocrinopathy in the nonobese diabetic mouse. Scand J Immunol 2004;60: $552-565$.

31 Peters MF, Ross CA. Isolation of a $40-\mathrm{kDa}$ Huntingtinassociated protein. J Biol Chem 2001;276:3188-3194 (E-pub 2000 October 3116).

32 Ongwijitwat S, Wong-Riley MT. Functional analysis of the rat cytochrome $\mathrm{C}$ oxidase subunit 6A1 promoter in primary neurons. Gene 2004;337: 163-171.

33 Rodriguez-Viciana $\mathrm{P}$, Warne $\mathrm{PH}$, Khwaja A, et al. Role of phosphoinositide 3-OH kinase in cell transformation and control of the actin cytoskeleton by Ras. Cell 1997;89:457-467.

34 Lee HY, Srinivas H, Xia D, et al. Evidence that phosphatidylinositol 3-kinase- and mitogen-activated protein kinase kinase-4/c-Jun NH2-terminal kinasedependent Pathways cooperate to maintain lung cancer cell survival. J Biol Chem 2003;278: 23630-23638 (E-pub 22003 April 23624).

35 Qian Y, Zhong X, Flynn DC, et al. ILK mediates actin filament rearrangements and cell migration and invasion through PI3K/Akt/Rac1 signaling. Oncogene 2005; 24:3154-3165.

36 Uhlik MT, Abell AN, Cuevas BD, et al. Wiring diagrams of MAPK regulation by MEKK1, 2, and 3 . Biochem Cell Biol 2004;82:658-663.

37 Natale DR, Watson AJ. Rac-1 and IQGAP are potential regulators of E-cadherin-catenin interactions during murine preimplantation development. Gene Expr Patterns 2002;2:17-22.
38 Bryce PJ, Oyoshi MK, Kawamoto S, et al. TRAF1 regulates Th2 differentiation, allergic inflammation and nuclear localization of the Th2 transcription factor, NIP45. Int Immunol 2006;18:101-111 (E-pub 2005 December 2013).

39 Gao J, Killedar S, Cornelius JG, et al. Sjogren's syndrome in the NOD mouse model is an interleukin-4 time-dependent, antibody isotype-specific autoimmune disease. J Autoimmun 2006;26:90-103 (E-pub 2006 January 2018).

40 Hacker H, Redecke V, Blagoev B, et al. Specificity in Toll-like receptor signalling through distinct effector functions of TRAF3 and TRAF6. Nature 2006;439: 204-207 (E-pub 2005 November 2023).

41 Ni CZ, Oganesyan G, Welsh K, et al. Key molecular contacts promote recognition of the BAFF receptor by TNF receptor-associated factor 3: implications for intracellular signaling regulation. J Immunol 2004;173: 7394-7400.

42 Hatzoglou A, Roussel J, Bourgeade MF, et al. TNF receptor family member BCMA (B cell maturation) associates with TNF receptor-associated factor (TRAF) 1, TRAF2, and TRAF3 and activates NF-kappa B, elk-1, c-Jun N-terminal kinase, and p38 mitogen-activated protein kinase. J Immunol 2000;165:1322-1330.

43 Yamamoto H, Kishimoto T, Minamoto S. NF-kappaB activation in CD27 signaling: involvement of TNF receptor-associated factors in its signaling and identification of functional region of CD27. J Immunol 1998; 161:4753-4759.

44 Hauer J, Puschner S, Ramakrishnan $\mathrm{P}$, et al. TNF receptor (TNFR)-associated factor (TRAF) 3 serves as an inhibitor of TRAF2/5-mediated activation of the noncanonical NF-kappaB pathway by TRAF-binding TNFRs. Proc Natl Acad Sci USA 2005;102:2874-2879 (E-pub 2005 February 2811).

45 Schneider P. The role of APRIL and BAFF in lymphocyte activation. Curr Opin Immunol 2005;17: 282-289.

46 Lavie F, Miceli-Richard C, Quillard J, et al. Expression of BAFF (BLyS) in T cells infiltrating labial salivary glands from patients with Sjogren's syndrome. J Pathol 2004;202:496-502.

47 Mariette X, Roux S, Zhang J, et al. The level of BLyS (BAFF) correlates with the titre of autoantibodies in human Sjogren's syndrome. Ann Rheum Dis 2003;62: 168-171.

48 Hjelmervik TO, Petersen K, Jonassen I, et al. Gene expression profiling of minor salivary glands clearly distinguishes primary Sjogren's syndrome patients from healthy control subjects. Arthritis Rheum 2005;52:1534-1544.

49 Bobik A, Kalinina N. Tumor necrosis factor receptor and ligand superfamily family members TNFRSF14 and LIGHT: new players in human atherogenesis. Arterioscler Thromb Vasc Biol 2001;21: 1873-1875.

50 Kim WJ, Kang YJ, Koh EM, et al. LIGHT is involved in the pathogenesis of rheumatoid arthritis by inducing the expression of pro-inflammatory cytokines and MMP-9 in macrophages. Immunology 2005;114: $272-279$.

51 Azuma M, Aota K, Tamatani T, et al. Suppression of tumor necrosis factor alpha-induced matrix metalloproteinase 9 production in human salivary gland acinar cells by cepharanthine occurs via downregulation of nuclear factor kappaB: a possible therapeutic 
agent for preventing the destruction of the acinar structure in the salivary glands of Sjogren's syndrome patients. Arthritis Rheum 2002;46:1585-1594.

52 Teixeira MM, Gristwood RW, Cooper N, et al. Phosphodiesterase (PDE)4 inhibitors: anti-inflammatory drugs of the future? Trends Pharmacol Sci 1997;18: 164-171.
53 Jin SL, Lan L, Zoudilova M, et al. Specific role of phosphodiesterase 4B in lipopolysaccharide-induced signaling in mouse macrophages. J Immunol 2005;175: 1523-1531.

54 Serreze DV. Autoimmune diabetes results from genetic defects manifest by antigen presenting cells. FASEB J 1993;7:1092-1096.

Supplementary Information accompanies the paper on the Laboratory Investigation website (http:// www.nature.com/labinvest) 